\title{
THE STABILITY OF STRONG VISCOUS CONTACT DISCONTINUITY TO A FREE BOUNDARY PROBLEM FOR COMPRESSIBLE NAVIER-STOKES EQUATIONS
}

\author{
Tingting Zheng* \\ Computer and Message Science College, Fujian Agriculture and Forestry University, \\ Fuzhou 350001, P. R. China
}

\begin{abstract}
This paper is concerned with nonlinear stability of strong viscous contact discontinuity to a free boundary problem for the one-dimensional full compressible Navier-Stokes equations in half space $[0, \infty)$. For the case when the local stability of the contact discontinuities was first studied by [1], later generalized by [2], local stability of weak viscous contact discontinuity is well-established by [3 7], but for the global stability of the impermeable gas , fewer strong nonlinear wave stability results have been obtained excluding zero dissipation [8] or $\gamma \rightarrow 1$ gas see [9]. Our main purpose is to deduce the corresponding nonlinear stability result by exploiting the elementary energy method. We will show in this paper that with a certain class of big perturbation the global stability result of strong viscous contact discontinuity to Navier-Stokes equations can be obtained.
\end{abstract}

AMS Subject Classifications (2000). 35B40, 35B45, 76N10,76N17

Keywords: Viscous contact discontinuity, Free boundary problem, Navier-Stokes equations

\section{Introduction}

This paper is concerned with a free boundary problem for a one-dimensional compressible viscous heat-conducting flow in the half space $\mathbb{R}_{+}=[0, \infty)$, which is governed by the following initialboundary value problem in Eulerian coordinate $(\tilde{x}, t)$ :

$$
\left\{\begin{array}{l}
\tilde{\rho}_{t}+(\tilde{\rho} \tilde{u})_{\tilde{x}}=0, \quad(\tilde{x}, t) \in \mathbb{R}_{+} \times \mathbb{R}_{+}, \\
(\tilde{\rho} \tilde{u})_{t}+\left(\tilde{\rho} \tilde{u}^{2}+\tilde{p}\right)_{\tilde{x}}=\mu \tilde{u} \tilde{x} \tilde{x}, \\
\left(\tilde{\rho}\left(\tilde{e}+\frac{\tilde{u}^{2}}{2}\right)\right)_{t}+\left(\tilde{\rho} \tilde{u}\left(\tilde{e}+\frac{\tilde{u}^{2}}{2}\right)+\tilde{p} \tilde{u}\right)_{\tilde{x}}=\kappa \tilde{\theta}_{\tilde{x} \tilde{x}}+\left(\mu \tilde{u} \tilde{u}_{\tilde{x}}\right)_{\tilde{x}},
\end{array}\right.
$$

where $\tilde{\rho}, \tilde{u}$ and $\tilde{\theta}$ are the density, the velocity and the absolute temperature, respectively, while $\mu>0$ is the viscosity coefficient and $\kappa>0$ is the heat-conductivity coefficients, respectively. The pressure $p=\tilde{p}(\tilde{\rho}, \tilde{\theta})$ are related by the second law of thermodynamics. To simplify our problem, we focus our attention on the perfect gas. In this situation

$$
\tilde{p}(\tilde{\rho}, \tilde{\theta})=R \tilde{\theta} \tilde{\rho}
$$

\footnotetext{
${ }^{*}$ Corresponding author, e-mail: asting16@sohu.com
} 


$$
\tilde{e}(\tilde{\rho}, \tilde{\theta})=\frac{R}{\gamma-1} \tilde{\theta}+\text { const }
$$

where $R>0$ is the gas constant and $\gamma>1$ is the adiabatic exponent. We consider the system (1.1) in the part $\tilde{x}>\tilde{x}(t)$, where $\tilde{x}=\tilde{x}(t)$ is a free boundary, with the following boundary condition

$$
\frac{d \tilde{x}(t)}{d t}=\tilde{u}(\tilde{x}(t), t), \tilde{x}(0)=0, \tilde{\theta}(\tilde{x}(t), t)=\theta_{-}>0,
$$

and

$$
\left.\left(\tilde{p}-\mu \tilde{u}_{\tilde{x}}\right)\right|_{\tilde{x}=\tilde{x}(t)}=p_{-},
$$

which means the gas is attached at the free boundary $\tilde{x}=\tilde{x}(t)$ to the atmosphere with pressure $p_{-}$and the initial data

$$
(\tilde{\rho}, \tilde{u}, \tilde{\theta})(\tilde{x}, 0)=\left(\tilde{\rho}_{0}, \tilde{u}_{0}, \tilde{\theta}_{0}\right)(\tilde{x}), \lim _{\tilde{x} \rightarrow+\infty}\left(\tilde{\rho}_{0}, \tilde{u}_{0}, \tilde{\theta}_{0}\right)(\tilde{x})=\left(\rho_{+}, 0, \theta_{+}\right),
$$

where $\rho_{+}, \theta_{+}$are positive constants and $\theta_{0}(0)=\theta_{-}$. Because here we only consider the case of a single contact discontinuity, we require

$$
p_{-}=p_{+}=R \theta_{+} \rho_{+}
$$

Since the boundary condition (1.3) means the particles always stay on the free boundary $\tilde{x}=\tilde{x}(t)$, if we use Lagrangian coordinates, then the free boundary becomes a fixed boundary. That is

$$
\left\{\begin{array}{l}
v_{t}-u_{x}=0, \quad(x, t) \in \mathbb{R}_{+} \times \mathbb{R}_{+}, \\
u_{t}+\left(\frac{R \theta}{v}\right)_{x}=\mu\left(\frac{u_{x}}{v}\right)_{x} \\
\frac{R}{\gamma-1} \theta_{t}+R \frac{\theta}{v} u_{x}=\kappa\left(\frac{\theta_{x}}{v}\right)_{x}+\mu \frac{u_{x}^{2}}{v} \\
\left.\theta\right|_{x=0}=\theta_{-}, \quad t>0, \\
\left(\frac{R \theta_{-}}{v}-\mu \frac{u_{x}}{v}\right)(0, t)=p_{+}, \quad t>0, \\
\left.(v, u, \theta)\right|_{t=0}=\left(v_{0}, u_{0}, \theta_{0}\right) \rightarrow\left(v_{+}, 0, \theta_{+}\right) \quad \text { as } \quad x \rightarrow+\infty
\end{array}\right.
$$

where $v_{+}$and $\theta_{ \pm}$are given positive constants, and $v_{0}, \theta_{0}>0$. In fact $v=1 / \rho(x, t), u=$ $u(x, t), \theta=\theta(x, t)$ and $R \theta / v=p(v, \theta)$ are the specific volume, velocity, temperature and pressure as in (1.1).

In terms of various boundary values, Matsumura 10] classified all possible large-time behaviors of the solutions for the one-dimensional (isentropic)compressible Navier-Stokes equations. In the case that $u(0, t)=0$ (resp. $u(0, t)<0$ ), the problem is called the impermeable wall (resp. outflow) problem in which the boundary condition of density can't be imposed. There have been a lot of works on the asymptotic behaviors of solutions to the initial-boundary value (or Cauchy) problem for the Navier-Stokes equations toward these basic waves or their viscous versions, see, for example, [3-25] and the references therein.

On the other hand, the problem of stability of contact discontinuities are associated with linear degenerate fields and are less stable than the nonlinear waves for the inviscid system (Euler equations). It was observed in [1,2], where the metastability of contact waves was studied 
for viscous conservation laws with artificial viscosity, that the contact discontinuity cannot be the asymptotic state for the viscous system, and a diffusive wave, which approximated the contact discontinuity on any finite time interval, actually dominates the large-time behavior of solutions. The nonlinear stability of contact discontinuity for the (full) compressible NavierStokes equations was then investigated in [3,6] for the free boundary value problem and [4,5] for the Cauchy problem.

As it is shown in the references, we construct viscous contact wave with artificial viscousity by the corresponding Euler system of (1.6) with Riemann initial data which reads as follows:

$$
\left\{\begin{array}{l}
v_{t}-u_{x}=0, \\
u_{t}+p(v, \theta)_{x}=0, \\
\frac{R}{\gamma-1} \theta_{t}+R \frac{\theta}{v} u_{x}=0, \\
(v, u, \theta)(x, 0)=\left(v_{-}, 0, \theta_{-}\right) \quad \text { if } \quad x<0, \\
(v, u, \theta)(x, 0)=\left(v_{+}, 0, \theta_{+}\right) \quad \text { if } \quad x>0 .
\end{array}\right.
$$

Because the corresponding Euler equations (1.7) with the Riemann initial data has the following soluitons

$$
(\bar{V}, \bar{U}, \bar{\Theta})= \begin{cases}\left(v_{-}, 0, \theta_{-}\right), & x<0, \\ \left(v_{+}, 0, \theta_{+}\right), & x>0\end{cases}
$$

provided that

$$
p_{-}=R \frac{\theta_{-}}{v_{-}}=p_{+}=R \frac{\theta_{+}}{v_{+}},
$$

as that in [3] we conjecture that the asymptotic limit $(V, U, \Theta)$ of (1.6) is as follows

$$
P(V, \Theta)=R \frac{\Theta}{V}=p_{+}, \quad U(x, t)=\frac{\kappa(\gamma-1) \Theta_{x}}{\gamma R \Theta},
$$

and $\Theta$ is the solution of the following problem

$$
\left\{\begin{array}{l}
\Theta_{t}=a(\ln \Theta)_{x x}, \quad a=\frac{\kappa p_{+}(\gamma-1)}{\gamma R^{2}}>0 \\
\Theta(0, t)=\theta_{-}, \\
\Theta(x, 0)=\Theta_{0} \rightarrow \theta_{+}, \quad \text { as } x \rightarrow+\infty
\end{array}\right.
$$

with $\Theta_{0}=\theta_{+}-\left(\theta_{+}-\theta_{-}\right) \exp \left\{1-(1+\alpha x)^{\delta_{0}}\right\}$. It is easy to check that there exist positive constant $M_{0}$ which is independent of $\delta_{0}$ and $\alpha$ such that

$$
\begin{aligned}
& \left\|\Theta_{0}-\theta_{+}\right\|_{L^{1}} \leq M_{0} \alpha^{-1} \sum_{n=0}^{\left[\frac{1}{\delta_{0}}\right]-1} \prod_{i=0}^{n}\left(\frac{1}{\delta_{0}}-i\right) \\
& \left|\Theta_{0 x}\right| \leq M_{0} \alpha \delta_{0}, \\
& \left|\Theta_{0 x x}\right| \leq M_{0} \alpha^{2} \delta_{0}, \\
& \left\|\Theta_{0 x}\right\|^{2} \leq M_{0} \alpha \delta_{0}, \\
& \left\|\Theta_{0 x}\right\|_{L^{1}\left(\mathbb{R}_{+}\right)} \leq M_{0}
\end{aligned}
$$




$$
\begin{aligned}
& \left\|\Theta_{0 x x}\right\|^{2}+\left\|\left(\ln \Theta_{0}\right)_{x x}\right\|^{2} \leq M_{0} \alpha^{3} \delta_{0}^{2}, \\
& \left\|\Theta_{0 x x x}\right\|^{2}+\left\|\left(\ln \Theta_{0}\right)_{x x x}\right\|^{2} \leq M_{0} \alpha^{5}, \\
& \int_{\mathbb{R}_{+}} \Theta_{0 x}^{2}(1+\alpha x) d x \leq M_{0} \alpha \delta_{0},
\end{aligned}
$$

here and following $\alpha$ is a positive constant which will be determined in Lemma 2.3 due to the artificial viscosity and $\delta_{0}$ is a small positive constant which is independent of $\theta_{ \pm}$. To sum up, we have constructed a pair of functions $(V, U, \Theta)$ such that

$$
\left\{\begin{array}{l}
R \frac{\Theta}{V}=p_{+}, \\
V_{t}=U_{x} \\
U_{t}+P(V, \Theta)_{x}=\mu\left(\frac{U_{x}}{V}\right)_{x}+F \\
\frac{R}{\gamma-1} \Theta_{t}+R \frac{\Theta}{V} U_{x}=\kappa\left(\frac{\Theta_{x}}{V}\right)_{x}+\mu \frac{U_{x}^{2}}{V}+G \\
(V, U, \Theta)(0, t)=\left(v_{-},\left.\frac{\kappa(\gamma-1)}{\gamma R} \frac{\Theta_{x}}{\Theta}\right|_{x=0}, \theta_{-}\right), \\
(V, U, \Theta)(x, 0)=\left(V_{0}, U_{0}, \Theta_{0}\right)=\left(\frac{R}{p_{+}} \Theta_{0}, \frac{\kappa(\gamma-1)}{\gamma R} \frac{\Theta_{0 x}}{\Theta_{0}}, \Theta_{0}\right) \rightarrow\left(v_{+}, 0, \theta_{+}\right), \text {as } x \rightarrow+\infty
\end{array}\right.
$$

where

$$
\begin{aligned}
G & =-\mu \frac{U_{x}^{2}}{V}=O\left((\ln \Theta)_{x x}^{2}\right), \\
F(x, t) & =\frac{\kappa(\gamma-1)}{\gamma R}\left\{(\ln \Theta)_{x t}-\mu\left(\frac{(\ln \Theta)_{x x}}{V}\right)_{x}\right\} \\
& =\frac{\kappa a(\gamma-1)-\mu p_{+} \gamma}{R \gamma}\left(\frac{(\ln \Theta)_{x x}}{\Theta}\right)_{x} .
\end{aligned}
$$

We shall show in the next section that $(V, U, \Theta)$ approximates $(\bar{V}, \bar{U}, \bar{\Theta})$ in $L^{p}$ norm with $p \geq 1$ on any finite time interval as the heat conductivity $\kappa$ goes to zero. So, we call $(V, U, \Theta)$ the viscous contact wave for the Navier-Stokes system (1.6). The definition can be more precise according to whether $H\left(\mathbb{R}_{+}\right)$-norm of the initial perturbation $\left(\varphi_{0}(x), \psi_{0}(x), \zeta_{0}(x)\right)$ and (or) $\left|\theta_{+}-\theta_{-}\right|$big or not, the stability results are classified into global (or local) stability of strong (or weak ) viscous contact wave.

Our main purpose is to justify that the solution $(v, u, \theta)$ of the Navier-Stokes system (1.3) asymptotically tends to the strong viscous contact discontinuity $(V, U, \Theta)$. Roughly speaking, the main result is : "if the oscillation of temperature and density are not small, the viscous contact discontinuity is asymptotic stable". To deduce the desired nonlinear stability result by the elementary energy method as in [3,7, 9,11,12, it is sufficient to deduce certain uniform (with respect to the time variable $t$ ) energy type estimates on the solution $(v(x, t), u(x, t), \theta(x, t))$ and how to establish the Poincaré type inequality in Lemma 4.1 without the smallness of $\left|\theta_{+}-\theta_{-}\right|$ which the arguments employed in [3,7, 11,12] is to use both smallness $\left|\theta_{+}-\theta_{-}\right|$and $N(t)=$ $\sup \|(\varphi, \psi, \zeta)\|_{H^{1}}$ to overcome such difficulties. One of the key points in such an argument is $0 \leq \tau \leq t$

that, based on the a priori assumption that $\sup \|(\varphi, \psi, \zeta)\|_{H^{1}}(\tau)$ is sufficiently small, one can 
deduce a uniform lower and upper positive bounds on the specific volume $v(x, t)$ and temperature $\theta(x, t)$. With such a bound on $v$ and $\theta$ in hand, one can deduce a priori $H\left(\mathbb{R}_{+}\right)$energy type estimates on $(\varphi, \psi, \zeta)$ in terms of the initial perturbation $\left(\varphi_{0}, \psi_{0}, \zeta_{0}\right)$ provided that $\left|\theta_{+}-\theta_{-}\right|$ suitably small, so the stability of weak contact discontinuity can be obtained . In fact if $N(t)$ not small and the perturbation of $\left\|\left(\varphi_{0 x}, \psi_{0 x}, \zeta_{0 x}\right)\right\|_{L^{2}\left(\mathbb{R}_{+}\right)}$not small (see [9]), the combination of the analysis similar as above with the standard continuation argument, it also can obtain the upper and lower bounds of $(v, \theta)$, then that yields the global stability of strong viscous contact discontinuity for the one-dimensional compressible Navier-Stokes equations in the condition of $\gamma \rightarrow 1$. In all, after researching the references carefully we find it is important to get the uniform time estimates of the viscous contact discontinuity we constructed, then we can obtain the energy estimates we expected, then the upper and lower bounds of $(v, \theta)$ come out . So the global stability result can be obtained. It is easy to see that in such a result, for all $t \in \mathbb{R}_{+}$, Osc $\theta(t):=\sup _{x \in \mathbb{R}_{+}} \theta(x, t)-\inf _{x \in \mathbb{R}_{+}} \theta(x, t) \geq\left|\theta_{+}-\theta_{-}\right|$, the oscillation of the temperature $\theta(x, t)$ should not be sufficiently small when $\left|\theta_{+}-\theta_{-}\right|$is not small. Similarly, we can also obtain the oscillation of the density $\rho(x, t)$ should not be sufficiently small too .

Following the above analysis, the rest of this paper is out lined as follows. In section 2 we study the properties of the viscous continuity $(V, U, \Theta)$ in (1.6). In section 3, we reformulate the problem and give the precise statement of our main theorem. Finally, we complete the proof of the main result by the global a priori estimates established in section 1 .

Throughout this paper, we shall denote $H^{l}\left(\mathbb{R}_{+}\right)$the usual $l-t h$ order Sobolev space with the norm

$$
\|f\|_{l}=\left(\sum_{j=0}^{l}\left\|\partial_{x}^{j} f\right\|^{2}\right)^{1 / 2}, \quad\|\cdot\|:=\|\cdot\|_{L^{2}\left(\mathbb{R}_{+}\right)} .
$$

For simplicity, we also use $C$ or $C_{i}(i=1,2,3 \ldots .$.$) to denote the various positive generic constants.$ $C(z)$ stands for constant about $z$ and $\lim _{z \rightarrow 0} C(z)=0 . \epsilon$ and $\epsilon_{i}(i=1,2,3 \ldots .$.$) stand for a small$ positive constant in Cauchy-Schwarz inequality and $\partial_{x}^{i}=\frac{\partial^{i}}{\partial x^{i}}$.

\section{Preliminaries}

This section is devoted to study of the viscous contact discontinuity $(V, U, \Theta)$ in (1.13). To finish it, we construct a parabolic equation about $\theta_{2}$ which play an important role in the time estimates of $\partial_{x}^{i} \Theta(i=1,2,3)$, it is shown as follows.

Lemma 2.1 If $\delta_{0}$ and $\Theta_{0}$ satisfying the condition in Theorem 3.1 and

$$
\theta_{2}(x, t)=\int_{0}^{+\infty}(4 \pi a t)^{-1 / 2}\left(\Theta_{0}(h)-\theta_{-}\right)\left\{\exp \left\{-\frac{(h-x)^{2}}{4 a t}\right\}-\exp \left\{-\frac{(h+x)^{2}}{4 a t}\right\}\right\} d h+\theta_{-},
$$

we can get

$$
\begin{aligned}
& \theta_{2 t}=a \theta_{2 x x} ; \\
& \theta_{2}(0, t)=\theta_{-} ; \\
& \theta_{2}(x, 0)=\theta_{20}(x)= \begin{cases}\Theta_{0}(x) \rightarrow \theta_{+}, & x>0 ; \\
-\Theta_{0}(-x)+2 \theta_{-} \rightarrow 2 \theta_{-}-\theta_{+}, & x \leq 0,\end{cases}
\end{aligned}
$$


and

$$
\int_{0}^{t}\left\|\theta_{2 x}\right\|^{2} d t \leq C(1+t)^{1 / 2}
$$

Proof. Because $\theta_{2}(x, t)$ can be rewrite to

$$
\theta_{2}(x, t)=\int_{-\infty}^{+\infty}(4 \pi a t)^{-1 / 2} \theta_{20}(h) \exp \left\{-\frac{(x-h)^{2}}{4 a t}\right\} d h,
$$

and $\theta_{20}(x) \in C^{1}(\mathbb{R})$, we find that $\theta_{2}(x, t)$ is a fundamental solution of (2.1) , it is easy to check $\lim _{t \rightarrow 0} \theta_{2}(x, t)=\theta_{20}(x)$, so we finish (2.1) .

Because

$$
\begin{aligned}
\theta_{2 x}= & \int_{0}^{+\infty}(4 \pi a t)^{-1 / 2}\left(\Theta_{0}(z)-\theta_{-}\right) \exp \left\{-\frac{(z-x)^{2}}{4 a t}\right\} \frac{z-x}{2 a t} d z \\
& +\int_{0}^{+\infty}(4 \pi a t)^{-1 / 2}\left(\Theta_{0}(z)-\theta_{-}\right) \exp \left\{-\frac{(z+x)^{2}}{4 a t}\right\} \frac{z+x}{2 a t} d z \\
= & \int_{0}^{\infty}(4 \pi a t)^{-1 / 2} \Theta_{0 z}(z) \exp \left\{\frac{-(z-x)^{2}}{4 a t}\right\} d z \\
& \quad-\int_{0}^{\infty}(4 \pi a t)^{-1 / 2} \Theta_{0 z}(z) \exp \left\{\frac{-(z+x)^{2}}{4 a t}\right\} d z,
\end{aligned}
$$

we use Hölder inequality and Fubini Theorem and $\left|\theta_{+}-\theta_{-}\right| \leq\left\|\Theta_{0 z}\right\|_{L^{1}\left(\mathbb{R}_{+}\right)}<C$ to (2.3) , then we can get

$$
\begin{aligned}
\int_{0}^{t} \int_{0}^{\infty} \theta_{2 x}^{2} d x d t \leq & C \int_{0}^{t} \int_{0}^{\infty}(4 \pi a t)^{-1}\left(\int_{0}^{\infty} \Theta_{0 z}\left(\exp \left\{-\frac{(z-x)^{2}}{4 a t}\right\}-\exp \left\{-\frac{(z+x)^{2}}{4 a t}\right\}\right) d z\right)^{2} d x d t \\
\leq & C \int_{0}^{t} \int_{0}^{\infty}(4 \pi a t)^{-1} \int_{0}^{\infty}\left|\Theta_{0 z}\right| \exp \left\{-\frac{(z-x)^{2}}{4 a t}\right\} d z d x \int_{0}^{\infty}\left|\Theta_{0 z}\right| d z d t \\
& \quad+C \int_{0}^{t} \int_{0}^{\infty}(4 \pi a t)^{-1} \int_{0}^{\infty}\left|\Theta_{0 z}\right| \exp \left\{-\frac{(z+x)^{2}}{4 a t}\right\} d z d x \int_{0}^{\infty}\left|\Theta_{0 z}\right| d z d t \\
\leq & C \sqrt{1+t}
\end{aligned}
$$

So we finish this lemma.

Now let's consider the time estimates of $\partial_{x}^{i} \Theta(i=1,2,3)$ of (1.11), we have the following results.

Lemma 2.2 If $\Theta_{0 x}$ satisfying the condition of (1.12) and a positive constant $M_{0}$ is independent of $\delta_{0}$ and $\alpha$, there exist a positive constant $C$ such that

$$
\begin{aligned}
& \left\|(\ln \Theta)_{x}\right\|^{2}+a \int_{0}^{t}\left\|(\ln \Theta)_{x x}\right\|^{2} d t \leq M_{0} \alpha \delta_{0} . \\
& (\operatorname{see}(2.12)-(2.13)) \\
& \left\|\Theta-\theta_{2}\right\|^{2}+\int_{0}^{t}\left\|(\ln \Theta)_{x}\right\|^{2} d t \leq C(1+t)^{1 / 2} . \\
& (\operatorname{see}(2.14)-2.15) \\
& \left\|(\ln \Theta)_{x}\right\|^{2} \leq C(1+t)^{-1 / 2} .
\end{aligned}
$$




$$
\begin{aligned}
& (\operatorname{see} 2.16-2.19) \\
& \left\|(\ln \Theta)_{x x}\right\|^{2} \leq C(1+t)^{-3 / 2} \text {. } \\
& \text { (see 2.20-2.24) } \\
& \left\|(\ln \Theta)_{x x}\right\|^{2}(1+t)+\int_{0}^{t}\left\|\partial_{x}^{3} \ln \Theta\right\|^{2}(1+t) d t \leq C \delta_{0}^{2} . \\
& \text { ( } \operatorname{see} 2.25) \\
& \left\|\partial_{x}^{3} \ln \Theta\right\|^{2} \leq C(1+t)^{-5 / 2} . \\
& \text { (see2.26-2.28) } \\
& \int_{\mathbb{R}_{+}} \Theta_{x}^{2} x d x \leq C \delta_{0} \text {. } \\
& \text { (see 2.29)-2.31) }
\end{aligned}
$$

Proof. From (1.11) we know

$$
(\ln \Theta)_{t}=a \frac{(\ln \Theta)_{x x}}{\Theta},
$$

both side of it multiply by $(\ln \Theta)_{x x}$ and integrate in $\mathbb{R}_{+} \times(0, t)$ we can get

$$
\begin{aligned}
& \left\|(\ln \Theta)_{x}\right\|^{2}+a \int_{0}^{t}\left\|(\ln \Theta)_{x x}\right\|^{2} d t \\
& \leq C\left\|\left(\ln \Theta_{0}\right)_{x}\right\|^{2}+\left.\int_{0}^{t}(\ln \Theta)_{t}(\ln \Theta)_{x}\right|_{0} ^{\infty} d t .
\end{aligned}
$$

So we can get

$$
\left\|(\ln \Theta)_{x}\right\|^{2}+a \int_{0}^{t}\left\|(\ln \Theta)_{x x}\right\|^{2} d t \leq M_{0} \alpha \delta_{0}
$$

Then if $\int_{0}^{t} \int_{\mathbb{R}_{+}}\left((\underline{1.11})_{1}-(\underline{2.1})_{1}\right) \times\left(\Theta-\theta_{2}\right) d x d t$ combine with Cauchy-Schwarz inequality we can get

$$
\left\|\Theta-\theta_{2}\right\|^{2}+\int_{0}^{t}\left\|(\ln \Theta)_{x}\right\|^{2} d t \leq C \int_{0}^{t}\left\|\theta_{2 x}\right\|^{2} d t
$$

Use (2.2) to (2.14) we can get

$$
\left\|\Theta-\theta_{2}\right\|^{2}+\int_{0}^{t}\left\|(\ln \Theta)_{x}\right\|^{2} d t \leq C(1+t)^{1 / 2} .
$$

That is (2.5).

Next, from

$$
\int_{0}^{t} \int_{\mathbb{R}_{+}}(1.11)_{1} \times \Theta^{-1}(\ln \Theta)_{x x}(1+t) d x d t
$$

we can get

$$
\int_{0}^{t}(1+t)\left((\ln \Theta)_{t}(\ln \Theta)_{x}\right)(0, t) d t
$$




$$
=a \int_{0}^{t} \int_{0}^{\infty} \frac{(\ln \Theta)_{x x}^{2}}{\Theta}(1+t) d x d t+\int_{0}^{t} \int_{0}^{\infty}\left((\ln \Theta)_{x}^{2}\right)_{t}(1+t) d x d t
$$

Because

$$
\int_{0}^{t}(1+t)(\ln \Theta)_{t}(\ln \Theta)_{x}(0, t) d t=0
$$

we can get

$$
\begin{aligned}
& (1+t)\left\|(\ln \Theta)_{x}\right\|^{2}+\int_{0}^{t} \int_{0}^{\infty}(1+t)(\ln \Theta)_{x x}^{2} d x d t \\
& \leq C\left\|\Theta_{0 x}\right\|^{2}+\int_{0}^{t} \int_{0}^{\infty}(\ln \Theta)_{x}^{2} d x d t .
\end{aligned}
$$

Combine with (2.15) we can get

$$
\begin{aligned}
& (1+t)\left\|(\ln \Theta)_{x}\right\|^{2}+\int_{0}^{t} \int_{0}^{\infty}(1+t)(\ln \Theta)_{x x}^{2} d x d t \\
& \leq C(1+t)^{1 / 2} .
\end{aligned}
$$

That means $\left\|(\ln \Theta)_{x}\right\|^{2} \leq C(1+t)^{-1 / 2}$, which is(2.6) .

Again from (1.11) 1 we can get

$$
(\ln \Theta)_{x t}=a\left(\frac{(\ln \Theta)_{x x}}{\Theta}\right)_{x} .
$$

Both side of (2.20) multiply $\partial_{x}^{3} \ln \Theta$ and get

$$
\left((\ln \Theta)_{x t} \partial_{x}^{2}(\ln \Theta)\right)_{x}-1 / 2\left(\partial_{x}^{2} \ln \Theta\right)_{t}=a\left(\frac{(\ln \Theta)_{x x}}{\Theta}\right)_{x} \partial_{x}^{3}(\ln \Theta) .
$$

Because

$$
\begin{aligned}
& \left((\ln \Theta)_{x t} \partial_{x}^{2}(\ln \Theta)\right)_{x}(1+t)^{2} \\
& =\left((\ln \Theta)_{x t}(\ln \Theta)_{x x}\right)_{x}(1+t)^{2} \\
& =a^{-1}\left((\ln \Theta)_{x t} \Theta_{t}\right)_{x}(1+t)^{2}
\end{aligned}
$$

then both side of (2.21) multiply $(1+t)^{2}$ then integrate in $\mathbb{R}_{+} \times(0, t)$ and combine with $\Theta_{t}(0, t)=$ $0, \Theta_{t}(\infty, t)=0, \Theta_{x}(\infty, t)=0$ and Cauchy-Schwarz inequality to get for some small $\epsilon>0$ we have

$$
\begin{aligned}
0 & \geq a \int_{0}^{t} \int_{0}^{\infty} \frac{(\ln \Theta)_{x x x}^{2}}{\Theta}(1+t)^{2} d x d t \\
& -\epsilon \int_{0}^{t} \int_{0}^{\infty}(1+t)^{2}(\ln \Theta)_{x x x}^{2} d x d t-C \epsilon^{-1} a \int_{0}^{t} \int_{0}^{\infty}(1+t)^{2}(\ln \Theta)_{x x}^{2}(\ln \Theta)_{x}^{2} d x d t \\
& +1 / 2\left\|(\ln \Theta)_{x x}\right\|^{2}(1+t)^{2}-1 / 2\left\|\left(\ln \Theta_{0}\right)_{x x}\right\|^{2}-\int_{0}^{t}\left\|(\ln \Theta)_{x x}\right\|^{2}(1+t) d x \\
\geq & C a \int_{0}^{t} \int_{0}^{\infty} \frac{(\ln \Theta)_{x x x}^{2}}{\Theta}(1+t)^{2} d x d t
\end{aligned}
$$




$$
\begin{aligned}
& -C \epsilon^{-1} a \int_{0}^{t} \int_{0}^{\infty}(1+t)^{2}\left\|(\ln \Theta)_{x x}\right\|\left\|(\ln \Theta)_{x x x}\right\|(\ln \Theta)_{x}^{2} d x d t \\
& +1 / 2\left\|(\ln \Theta)_{x x}\right\|^{2}(1+t)^{2}-1 / 2\left\|\left(\ln \Theta_{0}\right)_{x x}\right\|^{2}-\int_{0}^{t}\left\|(\ln \Theta)_{x x}\right\|^{2}(1+t) d x .
\end{aligned}
$$

Take (2.19) into (2.22) we can get

$$
\begin{aligned}
& \left\|(\ln \Theta)_{x x}\right\|^{2}(1+t)^{2}+\int_{0}^{t} \int_{0}^{\infty}(1+t)^{2}(\ln \Theta)_{x x x}^{2} d x d t \\
& \leq C(1+t)^{1 / 2}
\end{aligned}
$$

which also means

$$
\left\|(\ln \Theta)_{x x}\right\|^{2} \leq C(1+t)^{-3 / 2}
$$

and finish (2.7).

If both side of (2.21) multiply by $(1+t)$, similar as the proof of (2.23), when combine with (2.13) we can get

$$
\left\|(\ln \Theta)_{x x}\right\|^{2}(1+t)+\int_{0}^{t} \int_{0}^{\infty}(1+t)\left(\partial_{x}^{3} \ln \Theta\right)^{2} d x d t \leq C \delta_{0}^{2}
$$

which means (2.8).

From (2.20) we can get

$$
\partial_{t}(\ln \Theta)_{x x}=a \partial_{x}^{2}\left(\frac{(\ln \Theta)_{x x}}{\Theta}\right)
$$

Because

$$
\left((\ln \Theta)_{x x t}(\ln \Theta)_{x x x}\right)_{x}=\left(a^{-1} \Theta_{t t}(\ln \Theta)_{x x x}\right)_{x}
$$

when both side of (2.26) multiply $\partial_{x}^{4} \ln \Theta(1+\tau)^{3}$ then integrate in $\mathbb{R}_{+} \times(0, t)$ we can get

$$
\begin{aligned}
& \int_{0}^{t} \int_{0}^{\infty}\left(a^{-1} \Theta_{t t}(\ln \Theta)_{x x x}\right)_{x}(1+\tau)^{3} d x d \tau \\
& =\int_{0}^{t} \int_{0}^{\infty} a \partial_{x}^{2}\left(\frac{(\ln \Theta)_{x x}}{\Theta}\right) \partial_{x}^{4} \ln \Theta(1+\tau)^{3} d x d \tau \\
& \quad+\int_{0}^{t} \int_{0}^{\infty} \frac{1}{2}\left(\left(\partial_{x}^{3} \ln \Theta\right)^{2}\right)_{t}(1+\tau)^{3} d x d \tau .
\end{aligned}
$$

So use (2.19) and (2.23) we can get that for a small $\epsilon>0$, (2.27) can be change to

$$
\begin{aligned}
& \left\|\partial_{x}^{3} \ln \Theta\right\|^{2}(1+t)^{3}+C \int_{0}^{t}(1+\tau)^{3}\left\|\partial_{x}^{4} \ln \Theta\right\|^{2} d \tau \\
& \leq C+C \int_{0}^{t} \int_{0}^{\infty}\left(\partial_{x}^{3} \ln \Theta\right)^{2}(\ln \Theta)_{x}^{2}(1+\tau)^{3} d x d \tau+C \int_{0}^{t} \int_{0}^{\infty}(\ln \Theta)_{x x}^{4}(1+\tau)^{3} d x d \tau \\
& \quad+C \int_{0}^{t} \int_{0}^{\infty}\left(\partial_{x}^{2} \ln \Theta\right)^{2}(\ln \Theta)_{x}^{4}(1+\tau)^{3} d x d \tau+C \int_{0}^{t} \int_{0}^{\infty}\left(\partial_{x}^{3} \ln \Theta\right)^{2}(1+\tau)^{2} d x d \tau
\end{aligned}
$$




$$
\begin{aligned}
\leq & C \int_{0}^{t}\left\|(\ln \Theta)_{x}\right\|^{2}\left\|\partial_{x}^{3} \ln \Theta\right\|\left\|\partial_{x}^{4} \ln \Theta\right\|(1+\tau)^{3} d \tau+C \int_{0}^{t}\left\|(\ln \Theta)_{x x}\right\|^{3}\left\|\partial_{x}^{3} \ln \Theta\right\|(1+\tau)^{3} d \tau \\
& +\int_{0}^{t}\left\|(\ln \Theta)_{x x}\right\|^{4}\left\|(\ln \Theta)_{x}\right\|^{2}(1+\tau)^{3} d \tau+C(1+t)^{1 / 2} \\
\leq & \epsilon \int_{0}^{t}\left\|\partial_{x}^{4} \ln \Theta\right\|^{2}(1+\tau)^{3} d \tau+C \epsilon^{-1} \int_{0}^{t}\left\|\partial_{x}^{3} \ln \Theta\right\|^{2}(1+\tau)^{2} d \tau \\
& +C \epsilon^{-1} \int_{0}^{t}\left\|\partial_{x}^{2} \ln \Theta\right\|^{2}(1+\tau) d \tau+C(1+t)^{1 / 2} .
\end{aligned}
$$

Again using (2.19) and (2.23) we can get

$$
\left\|\partial_{x}^{3} \ln \Theta\right\|^{2}(1+t)^{3}+\int_{0}^{t}(1+\tau)^{3}\left\|\partial_{x}^{4} \ln \Theta\right\|^{2} d \tau \leq C(1+t)^{1 / 2} .
$$

This means (2.9) finished.

Now both side of (2.11) multiply by $(\ln \Theta)_{x x}(x-\beta \tau)(\beta>0)$ and integrate in $[\beta \tau, \infty) \times(0, t)$ we can get

$$
\begin{aligned}
\int_{0}^{t} & \int_{\beta \tau}^{\infty}\left((\ln \Theta)_{\tau}(\ln \Theta)_{x}(x-\beta \tau)\right)_{x} d x d \tau-1 / 2 \int_{0}^{t} \int_{\beta \tau}^{\infty}\left((\ln \Theta)_{x}^{2}(x-\beta \tau)\right)_{\tau} d x d \tau \\
& -a \int_{0}^{t} \int_{\beta \tau}^{\infty}(\ln \Theta)_{x x}(\ln \Theta)_{x} \Theta^{-1} d x d \tau-\frac{\beta}{2} \int_{0}^{t} \int_{\beta \tau}^{\infty}(\ln \Theta)_{x}^{2} d x d \tau \\
& -\int_{0}^{t} \int_{\beta \tau}^{\infty} a(\ln \Theta)_{x x}^{2}(x-\beta \tau) \Theta^{-1} d x d \tau=\sum_{i=1}^{5} K_{i}=0 .
\end{aligned}
$$

Use Cauchy-Schwarz inequality

$$
\left|K_{3}\right| \leq \frac{\beta}{4} \int_{0}^{t} \int_{\beta \tau}^{\infty}(\ln \Theta)_{x}^{2} d x d \tau+C \int_{0}^{t} \int_{\beta \tau}^{\infty}(\ln \Theta)_{x x}^{2} d x d \tau
$$

then combine with (2.4) we can get

$$
\left|K_{3}\right|+K_{4} \leq C \int_{0}^{t} \int_{\beta \tau}^{\infty}(\ln \Theta)_{x x}^{2} d x d \tau-\frac{\beta}{4} \int_{0}^{t} \int_{\beta \tau}^{\infty}(\ln \Theta)_{x}^{2} d x d \tau \leq C \alpha \delta_{0}-\frac{\beta}{4} \int_{0}^{t} \int_{\beta \tau}^{\infty}(\ln \Theta)_{x}^{2} d x d \tau .
$$

From $K_{1}$ to $K_{5}$, (2.29) can be change to

$$
\begin{aligned}
& \int_{\beta t}^{\infty}(\ln \Theta)_{x}^{2}(x-\beta t) d x+\int_{0}^{t} \int_{\beta \tau}^{\infty}(\ln \Theta)_{x x}^{2}(x-\beta \tau) d x d \tau \\
& +\frac{\beta}{4} \int_{0}^{t} \int_{\beta \tau}^{\infty}(\ln \Theta)_{x}^{2} d x d \tau \leq C \delta_{0} .
\end{aligned}
$$

Because

$$
\begin{aligned}
& \lim _{\beta \rightarrow 0}\left|\int_{\beta t}^{\infty}(\ln \Theta)_{x}^{2}(x-\beta t) d x-\int_{0}^{\infty}(\ln \Theta)_{x}^{2} x d x\right| \\
= & \lim _{\beta \rightarrow 0}\left|\int_{\beta t}^{\infty}(\ln \Theta)_{x}^{2}(x-\beta t-x) d x+\int_{0}^{\beta t}(\ln \Theta)_{x}^{2} x d x\right|=0,
\end{aligned}
$$


we can get

$$
0<\lim _{\beta \rightarrow 0} \int_{\beta t}^{\infty}(\ln \Theta)_{x}^{2}(x-\beta t) d x=\int_{0}^{\infty}(\ln \Theta)_{x}^{2} x d x \leq C \delta_{0} .
$$

So we finish this lemma.

The next lemma is concerned with the relation ship between the viscous continuity and the contact discontinuity. We shall show that as the heat conductivity $\kappa$ goes to zero, $(V, U, \Theta)$ will approximate $(\bar{V}, \bar{U}, \bar{\Theta})$ in $L^{p}\left(\mathbb{R}_{+}\right)(p \geq 1)$ norm on any finite time interval.

Lemma 2.3 For any given $T \in(0,+\infty)$ independent of $\kappa$ such that for any $p \geq 1$ and $t \in[0, T]$,

$$
\|(V-\bar{V}, U-\bar{U}, \Theta-\bar{\Theta})\|_{L^{p}\left(\mathbb{R}_{+}\right)} \rightarrow 0, \quad \text { as } \quad \kappa \rightarrow 0 .
$$

Proof. By the definition of $\bar{\Theta}$ in (1.8), to estimate $\|\Theta-\bar{\Theta}\|_{L^{p}\left(\mathbb{R}_{+}\right)}$, it suffices to prove

$$
\left\|\Theta-\theta_{+}\right\|_{L^{p}\left(\mathbb{R}_{+}\right)} \rightarrow 0, \text { as } \kappa \rightarrow 0, p \geq 1 .
$$

Because if $M_{0}>0$ is a constant independent of $\alpha$,

$$
\left\|\Theta-\theta_{+}\right\|_{L^{p}\left(\mathbb{R}_{+}\right)}^{p} \leq M_{0}\left\|\Theta-\theta_{+}\right\|_{L^{1}\left(\mathbb{R}_{+}\right)},
$$

the only thing we need to proof is

$$
\lim _{\kappa \rightarrow 0}\left\|\Theta-\theta_{+}\right\|_{L^{1}\left(\mathbb{R}_{+}\right)}=0 .
$$

In fact we set $\operatorname{sgn}_{\eta}(s)=\left\{\begin{array}{l}1, \quad s>\eta ; \\ s / \eta,-\eta \leq s \leq \eta ;, I_{\eta}(s)=\int_{0}^{s} \operatorname{sgn} \eta(s) d s \text { and } \eta>0 . \text { Both side } \\ -1, s<-\eta .\end{array}\right.$ of (1.11) 1 multiply by $\operatorname{sgn}_{\eta}\left(\Theta-\theta_{+}\right)$and integrate in $(0,+\infty) \times(0, t)$ we can get

$$
\begin{array}{r}
\int_{0}^{t}\left(\int_{0}^{+\infty} I_{\eta}\left(\Theta-\theta_{+}\right) d x\right)_{\tau} d \tau=-a \int_{0}^{t}(\ln \Theta)_{x}(0, t) \operatorname{sgn}_{\eta}\left(\Theta-\theta_{+}\right)(0, t) d \tau \\
-a \int_{0}^{t} \int_{0}^{+\infty}(\ln \Theta)_{x}^{2} \operatorname{sgn}_{\eta}^{\prime}\left(\Theta-\theta_{+}\right) d x d \tau .
\end{array}
$$

When $\eta \rightarrow 0$ and use (2.4), (2.8) we can get

$$
\begin{aligned}
& \left\|\Theta-\theta_{+}\right\|_{L^{1}\left(\mathbb{R}_{+}\right)}+a \int_{0}^{t} \int_{0}^{+\infty}(\ln \Theta)_{x}^{2} \operatorname{sgn}_{\eta}^{\prime}\left(\Theta-\theta_{+}\right) d x d \tau \\
& =a \int_{0}^{t}(\ln \Theta)_{x}(0, t) \operatorname{sgn}_{\eta}\left(\Theta-\theta_{-}\right)(0, t) d \tau+\left\|\Theta_{0}-\theta_{+}\right\|_{L^{1}\left(\mathbb{R}_{+}\right)} .
\end{aligned}
$$

Similar as (2.23), when we integrate (2.21) in $\mathbb{R}_{+} \times(0, t)$ and combine with (2.4) we can get that there exist constant $M_{0}>0$ independent of $\alpha$ such that

$$
\left\|(\ln \Theta)_{x x}\right\|^{2}+a \int_{0}^{t}\left\|\partial_{x}^{3}(\ln \Theta)\right\|^{2} d \tau \leq M_{0} \alpha^{3}+M_{0} a \alpha^{3}+M_{0} a^{-1} \alpha .
$$

Since $a=\kappa p_{+}(\gamma-1) /\left(\gamma R^{2}\right)$ and $\kappa \rightarrow 0$, we can choose $\alpha^{-1 / 2}=\kappa<1$, use (2.4) and (2.33) such that (2.32) is meant if constant $M_{0}>0$ is independent of $\alpha$, we have

$$
\left\|\Theta-\theta_{+}\right\|_{L^{1}\left(\mathbb{R}_{+}\right)} \leq M_{0} t\left((a \alpha)^{3 / 4}+a^{5 / 4} \alpha\right)+M_{0} \alpha^{-1} \leq M_{0} \kappa^{3 / 8}(t+1),
$$


so we get $\|(V-\bar{V}, \Theta-\bar{\Theta})\|_{L^{p}} \rightarrow 0$ as $\kappa \rightarrow 0$ with any $t \in[0, T]$.

It remains to estimate $\|U-\bar{U}\|_{L^{p}}$. To do so, both side of (2.20) multiply by $\operatorname{sgn}_{\eta}\left((\ln \Theta)_{x}\right)$ then integrate in $\mathbb{R}_{+} \times(0, t)$ we can get

$$
\begin{aligned}
& \int_{0}^{t}\left(\int_{\mathbb{R}_{+}} I_{\eta}\left((\ln \Theta)_{x}\right) d x\right)_{\tau} d \tau+a \int_{0}^{t} \int_{\mathbb{R}_{+}} \frac{(\ln \Theta)_{x x}^{2}}{\Theta} \operatorname{sgn}_{\eta}^{\prime}\left((\ln \Theta)_{x}\right) d x d \tau \\
& =-a \int_{0}^{t} \theta_{-}^{-1}(\ln \Theta)_{x x}(0, \tau) \operatorname{sgn}_{\eta}\left((\ln \Theta)_{x}\right)(0, \tau) d \tau \\
& =-\int_{0}^{t} \theta_{-}^{-1} \Theta_{\tau}(0, t) \operatorname{sgn}_{\eta}\left((\ln \Theta)_{x}\right)(0, \tau) d \tau=0 .
\end{aligned}
$$

Again let $\eta \rightarrow 0$ we can get that there exist constant $M_{0}>0$ independent of $\alpha$ such that

$$
\begin{aligned}
& \int_{\mathbb{R}_{+}}\left|(\ln \Theta)_{x}\right| d x+a \int_{0}^{t} \int_{\mathbb{R}_{+}} \frac{(\ln \Theta)_{x x}^{2}}{\Theta} \operatorname{sgn}_{\eta}^{\prime}\left((\ln \Theta)_{x}\right) d x d \tau \\
& \leq M_{0}
\end{aligned}
$$

Use the definition of $U$ in (1.10) and combine with (2.4), (2.33) and (2.34) we know that

$$
\begin{aligned}
& \|U-\bar{U}\|_{L^{p}}^{p} \leq M_{0} \kappa^{p}\left\|(\ln \Theta)_{x}\right\|_{L^{1}}\left\|(\ln \Theta)_{x}\right\|^{(p-1) / 2}\left\|(\ln \Theta)_{x x}\right\|^{(p-1) / 2} \\
& \leq M_{0} \kappa^{p} \alpha^{p-1} .
\end{aligned}
$$

Remind that $\alpha=\kappa^{-1 / 2}$, so we can get

$$
\lim _{\kappa \rightarrow 0}\|U-\bar{U}\|_{L^{p}}=0
$$

The proof of Lemma 2.3 is therefore complete, which also means $(V, U, \Theta)$ is viscous contact discontinuity. $\square$

\section{Reformulation and Main result}

Let $(v, u, \theta)$ be the solution of the problem (1.6), and let $(V, U, \Theta)$ be the viscous contact discontinuity constructed in (1.13). Denote

$$
\begin{aligned}
& \varphi(x, t)=v(x, t)-V(x, t), \\
& \psi(x, t)=u(x, t)-U(x, t), \\
& \zeta(x, t)=\theta(x, t)-\Theta(x, t) .
\end{aligned}
$$

Combining (1.13) and (1.6), the original problem can be reformulated as

$$
\left\{\begin{array}{l}
\varphi_{t}=\psi_{x}, \\
\psi_{t}-\left(\frac{R \Theta}{v V} \varphi\right)_{x}+\left(\frac{R \zeta}{v}\right)_{x}=-\mu\left(\frac{U_{x}}{v V} \varphi\right)_{x}+\mu\left(\frac{\psi_{x}}{v}\right)_{x}-F \\
\frac{R}{\gamma-1} \zeta_{t}+\frac{R \theta}{v}\left(\psi_{x}+U_{x}\right)-\frac{R \Theta}{V} U_{x}=\kappa\left(\frac{\zeta_{x}}{v}\right)_{x}-\kappa\left(\frac{\Theta_{x} \varphi}{v V}\right)_{x}+\mu\left(\frac{u_{x}^{2}}{v}-\frac{U_{x}^{2}}{V}\right)-G, \\
\zeta(0, t)=0, \\
\left(\frac{R \theta_{-}}{V+\varphi}-\mu \frac{U_{x}+\psi_{x}}{V+\varphi}\right)(0, t)=p_{+}, \\
(\varphi, \psi, \zeta)(x, 0)=\left(\varphi_{0}, \psi_{0}, \zeta_{0}\right)=\left(v_{0}-V_{0}, u_{0}-U_{0}, \theta_{0}-\Theta_{0}\right) .
\end{array}\right.
$$


From (1.13) it is easy to check that the initial-boundary data in (3.2) satisfies the compatible condition, and

$$
(\varphi, \psi, \zeta)(x, 0)=\left(\varphi_{0}, \psi_{0}, \zeta_{0}\right) \rightarrow(0,0,0) \text { as } x \rightarrow+\infty .
$$

To state our main result, we assume throughout of this section that

$$
\left(\varphi_{0}, \zeta_{0}\right)(x) \in H_{0}^{1}(0, \infty), \quad \psi_{0}(x) \in H^{1}(0, \infty) .
$$

Moreover, for an interval $I \in[0, \infty)$, we define the function space

$$
X(I)=\left\{(\varphi, \psi, \zeta) \in C\left(I, H^{1}\right) \mid \varphi_{x} \in L^{2}\left(I ; L^{2}\right),\left(\psi_{x}, \zeta_{x}\right) \in L^{2}\left(I ; H^{1}\right)\right\} .
$$

Our main results of this paper now reads as follows.

Theorem 3.1 There exist positive constants $C>1$ and $\eta_{0}$ such that if $\|\left(v_{0}-V_{0}, u_{0}-U_{0}, \theta_{0}-\right.$ $\left.\Theta_{0}\right)\left\|_{L^{2}} \leq \eta_{0},\right\|\left(v_{0 x}-V_{0 x}, u_{0 x}-U_{0 x}, \theta_{0 x}-\Theta_{0 x}\right) \|_{L^{2}} \leq C$, (3.2) has a unique global solution $(\varphi, \psi, \zeta)$ satisfying $(\varphi, \psi, \zeta) \in X([0, \infty))$ and

$$
\sup _{x \in \mathbb{R}_{+}}|(\varphi, \psi, \zeta)| \rightarrow 0, \text { as } t \rightarrow \infty .
$$

In this section, to study the asymptotic behavior of the solution to the free boundary problem (1.6), we will do some preparation lemmas and list some priori estimates which are important to the proof of Theorem 3.1 .

We shall prove Theorem 3.1 by combining the local existence and the global-in-time priori estimates. Since the local existence of the solution is well known (see, for example, [3]), we omit it here for brevity. to prove the global existence part of Theorem 3.1, it is sufficient to establish the following priori estimates.

Proposition 3.1 (A priori estimate) Let $(\varphi, \psi, \zeta) \in X([0, t])$ be a solution of problem (3.2) for some $t>0$. Then there exist positive constants $C\left(\delta_{0}\right)<1$ and $C$ which are all independent of $t$ and $(v, \theta)$, such that if $m \leq v, \theta \leq M$ and $1<N(t)=\sup _{0 \leq \tau \leq t}\|(\varphi, \psi, \zeta)\|_{1} \leq C$, it holds that

$$
\begin{aligned}
& \sup _{0 \leq \tau \leq t}\|(\psi, \varphi, \zeta)\|^{2}(t)+\int_{0}^{t}\left\|\left(\psi_{x}, \zeta_{x}\right)\right\|^{2}(\tau) d \tau \\
& \leq C\left\|\left(\varphi_{0}, \psi_{0}, \zeta_{0}\right)\right\|^{1 / 2}+C\left(\delta_{0}\right) . \\
& \sup _{0 \leq \tau \leq t}\left\|\left(\psi_{x}, \varphi_{x}, \zeta_{x}\right)\right\|^{2}(t)+\int_{0}^{t}\left(\left\|\varphi_{x}\right\|^{2}(\tau)+\left\|\left(\psi_{x}, \zeta_{x}\right)\right\|_{1}^{2}(\tau)\right) d \tau \\
& \leq C\left\|\left(\varphi_{0}, \psi_{0}, \zeta_{0}\right)\right\|_{1}+C\left(\delta_{0}\right) .
\end{aligned}
$$

\section{Proof of Theorem 3.1}

Under the preparations in last section, the main task here is to finish (3.3). This part we also do some preparations. we must use the results

$$
\begin{aligned}
& \left|V_{x}\right| \leq C\left|\Theta_{x}\right|, \\
& \left|\Theta_{x}\right|^{2} \leq C\left\|(\ln \Theta)_{x}\right\|\left\|(\ln \Theta)_{x x}\right\|, \\
& \left|U_{x}\right| \leq C\left|(\ln \Theta)_{x x}\right|,
\end{aligned}
$$




$$
\left|U_{x}\right|^{2} \leq C\left\|(\ln \Theta)_{x x}\right\|\left\|(\ln \Theta)_{x x x}\right\|,
$$

which follow from (1.10)-(1.13) . Also we set $C\left(\delta_{0}\right)$ stands for small constants about $\delta_{0}$, $\left\|\left(\varphi_{0}, \psi_{0}, \zeta_{0}\right)\right\|$ is asked suitably small, $C_{v}=\frac{R}{\gamma-1}$ and

$$
\epsilon_{1} \ll \epsilon_{2} \ll \epsilon_{3} .
$$

Before establishing (3.3), we first estimate the value of $\varphi(0, t)$ on the boundary $x=0$ by the boundary condition (3.2). Let $\varphi(t)=\varphi(0, t)$. Since $U_{x}(0, t)=V_{t}(0, t)=0$, the boundary condition of (3.2) yields

$$
\frac{R \theta_{-}}{v_{-}+\varphi(t)}-\mu \frac{\varphi_{t}(t)}{v_{-}+\varphi(t)}=p_{+}, t>0
$$

Direct computation gives

$$
\varphi_{t}(t)=-\frac{p_{+}}{\mu} \varphi(t), \varphi(0)=\varphi_{0}(0) .
$$

It follows then that

$$
\varphi(t)=\varphi_{0}(0) e^{-p_{+} t / \mu} .
$$

Lemma 4.1 If $C\left(\delta_{0}\right)>0$ is a small constant about $\delta_{0}$

$$
\int_{0}^{t} \int_{\mathbb{R}_{+}} \Theta_{x}^{2}\left(\varphi^{2}+\zeta^{2}\right) d x d \tau \leq C\left(\delta_{0}\right) \int_{0}^{t}\left\|\left(\varphi_{x}, \zeta_{x}\right)\right\|^{2} d \tau+C \varphi_{0}(0) .
$$

Proof. Because if $x>0$

$$
\begin{aligned}
& \frac{\varphi^{2}}{x+1}=\int_{0}^{x}\left(\frac{2 \varphi \varphi_{x}}{x+1}-\frac{\varphi^{2}}{(x+1)^{2}}\right) d x+\varphi^{2}(0, t) \\
& =\int_{0}^{x}\left(\varphi_{x}^{2}-\left(\varphi_{x}-\frac{\varphi}{x+1}\right)^{2}\right) d x+\varphi^{2}(0, t) \leq \int_{0}^{x} \varphi_{x}^{2} d x+C \varphi_{0}(0) e^{-p_{+} t / \mu} \leq\left\|\varphi_{x}\right\|^{2}+C \varphi_{0}(0) e^{-p_{+} t / \mu} .
\end{aligned}
$$

similar as above we can get

$$
\frac{\zeta^{2}}{x+1} \leq \int_{0}^{x} \zeta_{x}^{2} d x \leq\left\|\zeta_{x}\right\|^{2}
$$

As to

$$
\begin{aligned}
& \int_{0}^{t} \int_{0}^{+\infty} \Theta_{x}^{2}\left(\varphi^{2}+\zeta^{2}\right) d x d \tau \\
& \leq \int_{0}^{t} \int_{0}^{+\infty} \Theta_{x}^{2}(x+1) \frac{\left(\varphi^{2}+\zeta^{2}\right)}{1+x} d x d \tau \\
& \leq \int_{0}^{t}\left(\int_{0}^{+\infty} \Theta_{x}^{2}(1+x) d x\right)\left\|\left(\varphi_{x}, \zeta_{x}\right)\right\|^{2} d \tau+C \varphi_{0}(0),
\end{aligned}
$$

use (2.4) and (2.10) we can get

$$
\int_{0}^{t} \int_{\mathbb{R}_{+}} \Theta_{x}^{2}\left(\varphi^{2}+\zeta^{2}\right) d x d \tau \leq C\left(\delta_{0}\right) \int_{0}^{t}\left\|\left(\varphi_{x}, \zeta_{x}\right)\right\|^{2} d \tau+C \varphi_{0}(0),
$$

and we finish this lemma. $\square$

Now, let's finish (3.3) by the following lemmas. 
Lemma 4.2 If $\epsilon_{1}>0$ and $C\left(\delta_{0}\right)>0$ are small constant about $\delta_{0}$, we can get

$$
\begin{aligned}
& \int_{\mathbb{R}_{+}}\left(\varphi^{2}+\psi^{2}+\zeta^{2}\right) d x+\int_{0}^{t}\left\|\left(\psi_{x}, \zeta_{x}\right)\right\|^{2} d \tau \\
& \leq C\left(\delta_{0}\right)+C\left\{\epsilon_{1} \int_{0}^{t}\left\|\varphi_{x}\right\|^{2} d \tau+\left\|\left(\varphi_{0}, \psi_{0}, \zeta_{0}\right)\right\|^{2}\right\} .
\end{aligned}
$$

Proof. Set

$$
\begin{gathered}
\Phi(z)=z-\ln z-1, \\
\Psi(z)=z^{-1}+\ln z-1,
\end{gathered}
$$

where $\Phi^{\prime}(1)=\Phi(1)=0$ is a strictly convex function around $z=1$. Similar to the proof in [3], we deduce from (3.2) that

$$
\begin{aligned}
& \left(\frac{\psi^{2}}{2}+R \Theta \Phi\left(\frac{v}{V}\right)+C_{v} \Theta \Phi\left(\frac{\theta}{\Theta}\right)\right)_{t} \\
& \quad+\mu \frac{\Theta \psi_{x}^{2}}{v \theta}+\kappa \frac{\Theta \zeta_{x}^{2}}{v \theta^{2}}+H_{x}+Q=\mu\left(\frac{\psi \psi_{x}}{v}\right)_{x}-F \psi-\frac{\zeta G}{\theta}
\end{aligned}
$$

where

$$
H=R \frac{\zeta \psi}{v}-R \frac{\Theta \varphi \psi}{v V}+\mu \frac{U_{x} \varphi \psi}{v V}-\kappa \frac{\zeta \zeta_{x}}{v \theta}+\kappa \frac{\Theta_{x} \varphi \zeta}{v \theta V}
$$

and

$$
\begin{aligned}
Q= & p_{+} \Phi\left(\frac{V}{v}\right) U_{x}+\frac{p_{+}}{\gamma-1} \Phi\left(\frac{\Theta}{\theta}\right) U_{x}-\frac{\zeta}{\theta}\left(p_{+}-p\right) U_{x}-\mu \frac{U_{x} \varphi \psi_{x}}{v V} \\
& -\kappa \frac{\Theta_{x}}{v \theta^{2}} \zeta \zeta_{x}-\kappa \frac{\Theta \Theta_{x}}{v \theta^{2} V} \varphi \zeta_{x}-2 \mu \frac{U_{x}}{v \theta} \zeta \psi_{x}+\kappa \frac{\Theta_{x}^{2}}{v \theta^{2} V} \varphi \zeta+\mu \frac{U_{x}^{2}}{v \theta V} \varphi \zeta \\
= & \sum_{i=1}^{9} Q_{i} .
\end{aligned}
$$

Note that $p=R \theta / v, p_{+}=R \Theta / V$ and (1.10), use integrate by part and Cauchy-Schwarz inequality can get

$$
\begin{aligned}
Q_{1}+Q_{2}= & R a\left(\Phi\left(\frac{V}{v}\right)(\ln \Theta)_{x}\right)_{x}+\frac{R a}{\gamma-1}\left(\Phi\left(\frac{\Theta}{\theta}\right)(\ln \Theta)_{x}\right)_{x} \\
& -a R(\ln \Theta)_{x}\left(\frac{V \varphi_{x} \varphi-V_{x} \varphi^{2}}{V v^{2}}\right) \\
& -a \frac{p_{+}}{\gamma-1}(\ln \Theta)_{x}\left(\frac{\Theta \zeta_{x} \zeta-\Theta_{x} \zeta^{2}}{\Theta \theta^{2}}\right) \\
\geq & \left(p_{+} \Phi\left(\frac{V}{v}\right) U+\frac{p_{+}}{\gamma-1} \Phi\left(\frac{\Theta}{\theta}\right) U\right)_{x} \\
& -\epsilon\left(\zeta_{x}^{2}+\varphi_{x}^{2}\right)-C \epsilon^{-1} \Theta_{x}^{2}\left(\zeta^{2}+\varphi^{2}\right) .
\end{aligned}
$$

Similarly, using $p-p_{+}=\frac{R \zeta-p_{+} \varphi}{v}$, we can get

$$
Q_{3} \geq \frac{R \zeta-p_{+} \varphi}{v}\left(\frac{\zeta}{\theta} U_{x}\right) \geq\left(\frac{R \zeta^{2} U}{v \theta}-\frac{p_{+} \zeta \varphi U}{\theta v}\right)_{x}-\epsilon\left(\zeta_{x}^{2}+\varphi_{x}^{2}\right)-C \epsilon^{-1} \Theta_{x}^{2}\left(\zeta^{2}+\varphi^{2}\right) .
$$


And

$$
\begin{aligned}
\left(Q_{4}+Q_{7}\right)+\left(Q_{5}+Q_{6}+Q_{8}\right)+Q_{9} \geq & -C \epsilon^{-1}(\ln \Theta)_{x x}^{2}-\epsilon \psi_{x}^{2} \\
& -\epsilon \zeta_{x}^{2}-C \epsilon^{-1} \Theta_{x}^{2}\left(\zeta^{2}+\varphi^{2}\right) \\
& -C \epsilon^{-1}\left|(\ln \Theta)_{x x}\right|^{2}\left(\zeta^{2}+\varphi^{2}\right) .
\end{aligned}
$$

At the end we use the definition of $F$ and $G$ in (1.14) then combine with the general inequality skills as above to get

$$
\begin{aligned}
-F \psi-G \frac{\zeta}{\theta}=- & \frac{\kappa a(\gamma-1)-\mu p_{+} \gamma}{R \gamma}\left(\frac{(\ln \Theta)_{x x}}{\Theta}\right)_{x} \psi \\
& +\frac{\mu p_{+}}{R \Theta}\left(\frac{\kappa(\gamma-1)}{R \gamma}(\ln \Theta)_{x x}\right)^{2} \frac{\zeta}{\theta} \\
\leq- & \frac{\kappa a(\gamma-1)-\mu p_{+} \gamma}{R \gamma}\left(\frac{(\ln \Theta)_{x x}}{\Theta} \psi\right)_{x}+\frac{\kappa a(\gamma-1)-\mu p_{+} \gamma}{R \gamma} \frac{(\ln \Theta)_{x x}}{\Theta} \psi_{x} \\
& +\frac{\mu p_{+}}{R \Theta}\left(\frac{\kappa(\gamma-1)}{R \gamma}(\ln \Theta)_{x x}\right)^{2} \frac{\zeta}{\theta} \\
\leq- & \frac{\kappa a(\gamma-1)-\mu p_{+} \gamma}{R \gamma}\left(\frac{(\ln \Theta)_{x x}}{\Theta} \psi\right)_{x}+\epsilon \psi_{x}^{2}+C \epsilon^{-1}(\ln \Theta)_{x x}^{2} .
\end{aligned}
$$

Integrating (4.6) -(4.9) in $\mathbb{R} \times(0, t)$, using (2.4), (2.8) and the boundary condition about $(\varphi, \psi, \zeta)$ of (3.2), (4.4) and $\Theta_{t}(0, t)=a(\ln \Theta)_{x x}(0, t)=0, \Phi\left(\frac{\Theta}{\theta}\right)(0, t)=0$ to estimate the terms $\left(p_{+} \Phi\left(\frac{V}{v}\right) U+\frac{p_{+}}{\gamma-1} \Phi\left(\frac{\Theta}{\theta}\right) U\right)_{x}, \mu\left(\frac{\psi \psi_{x}}{v}\right)_{x},\left(\frac{(\ln \Theta)_{x x} \psi}{\Theta}\right)_{x}$ and $H_{x}$, we can know that because

$$
\begin{aligned}
& \int_{0}^{t}\left(\operatorname{Ra} \Phi\left(\frac{V}{v}\right)\left|(\ln \Theta)_{x}\right|+\mu\left|\frac{\psi \psi_{x}}{v}\right|+\left|\frac{(\ln \Theta)_{x x} \psi}{\Theta}\right|+|H|\right)(0, \tau) d \tau \\
& \leq C \int_{0}^{t}\left|(\ln \Theta)_{x}\right|(0, \tau)\left(|\varphi|(\tau)+\left|\varphi_{\tau}\right|(\tau)\right) d \tau \leq C\left(\delta_{0}\right),
\end{aligned}
$$

it is easy to get

$$
\begin{aligned}
& \left|\int_{0}^{t} \int_{\mathbb{R}_{+}}\left(\mu\left(p_{+} \Phi\left(\frac{V}{v}\right) U+\frac{p_{+}}{\gamma-1} \Phi\left(\frac{\Theta}{\theta}\right) U\right)_{x}+\left(\frac{(\ln \Theta)_{x x} \psi}{\Theta}\right)_{x}+H_{x}\right) d x d \tau\right| \\
& \leq C\left(\delta_{0}\right) .
\end{aligned}
$$

In the end from combine with the estimates from $Q_{1}$ to $Q_{9}$, (4.10) and Lemma 4.1 we have

$$
\begin{aligned}
& \int_{\mathbb{R}_{+}}\left(R \theta \Phi\left(\frac{v}{V}\right)+\frac{1}{2} \psi^{2}+C_{v} \theta \Phi\left(\frac{\theta}{\Theta}\right)\right) d x+\int_{0}^{t}\left\|\left(\psi_{x} /(\sqrt{v \theta}), \zeta_{x} /(\theta \sqrt{v})\right)\right\|^{2} d \tau \\
& \leq C \epsilon_{1}^{-1} \int_{0}^{t} \int_{0}^{\infty} \Theta_{x}^{2}\left(\varphi^{2}+\zeta^{2}\right) d x d \tau+C\left\{\epsilon_{1} \int_{0}^{t}\left\|\varphi_{x}\right\|^{2} d \tau+\left\|\left(\varphi_{0}, \psi_{0}, \zeta_{0}\right)\right\|^{2}\right\}+C\left(\delta_{0}\right) \\
& \leq C\left(\delta_{0}\right) \int_{0}^{t}\left\|\left(\psi_{x}, \zeta_{x}\right)\right\|^{2} d \tau++C\left\{\epsilon_{1} \int_{0}^{t}\left\|\varphi_{x}\right\|^{2} d \tau+\left\|\left(\varphi_{0}, \psi_{0}, \zeta_{0}\right)\right\|^{2}\right\}+C\left(\delta_{0}\right) .
\end{aligned}
$$

Use the condition of Proposition 3.1 which is $m \leq v, \theta \leq M$, we finish this lemma. 
Lemma 4.3 For a small $\epsilon_{2}>0, C\left(\delta_{0}\right)>0$ is a small constant about $\delta_{0}$, we have

$$
\begin{aligned}
& \|(\varphi, \psi, \zeta)\|^{2}+\left\|\left(\psi_{x}, \zeta_{x}\right)\right\|^{2}+\int_{0}^{t}\left\|\left(\psi_{x x}, \zeta_{x x}\right)\right\|^{2} d t \\
& \leq C\left(\left\|\left(\psi_{0 x}, \zeta_{0 x}\right)\right\|^{2}+\epsilon_{2}^{-1}\left\|\left(\varphi_{0}, \psi_{0}, \zeta_{0}\right)\right\|^{2}\right)+C \epsilon_{2}^{-1} \int_{0}^{t}\left\|\varphi_{x}\right\|^{2} d \tau+C\left(\delta_{0}\right)+C \varphi_{0}(0) .
\end{aligned}
$$

Proof. First to get the estimate of $\left\|\psi_{x}(t)\right\|$, multiply both side of $(\sqrt{3.2})_{2}$ to $\psi_{x x}$ to get

$$
\begin{aligned}
& \left(\frac{\psi_{x}^{2}}{2}\right)_{t}+\mu \frac{\psi_{x x}^{2}}{v}=\mu \frac{\psi_{x} v_{x}}{v^{2}} \psi_{x x}+\mu\left(\frac{U_{x} \varphi}{v V}\right)_{x} \psi_{x x} \\
& -R\left(\frac{\Theta \varphi}{v V}\right)_{x} \psi_{x x}+R\left(\frac{\zeta}{v}\right)_{x} \psi_{x x}+F \psi_{x x}+\left(\psi_{t} \psi_{x}\right)_{x}:=\sum_{i=1}^{6} I_{i} .
\end{aligned}
$$

use last inequality integrate in $\mathbb{R}_{+} \times(0, t)$

$$
\begin{aligned}
& \left\|\psi_{x}(t)\right\|^{2}+\int_{0}^{t}\left\|\psi_{x x}(\tau)\right\|^{2} d \tau \\
& \quad \leq C\left\|\psi_{0 x}\right\|^{2}+C \sum_{i=1}^{6}\left|\int_{0}^{t} \int_{0}^{\infty} I_{i} d x d \tau\right| .
\end{aligned}
$$

Now deal with $\iint\left|I_{i}\right| d x d \tau$ in the right side of (4.11). Using $\epsilon$ small and $v=\varphi+V$, $R \Theta / V=p_{+}$and (2.6), (2.7), (4.1) to get

$$
\begin{aligned}
& \int_{0}^{t} \int_{0}^{\infty}\left|I_{1}\right| d x d \tau \leq C \int_{0}^{t} \int_{0}^{\infty}\left|V_{x}\left\|\psi_{x}\right\| \psi_{x x}\right| d x d \tau+C \int_{0}^{t} \int_{0}^{\infty}\left|\varphi_{x}\left\|\psi_{x}\right\| \psi_{x x}\right| d x d \tau \\
& \leq C \int_{0}^{t}\left\|V_{x}\right\|\left\|\psi_{x}\right\|_{L^{\infty}}\left\|\psi_{x x}\right\| d \tau+C \int_{0}^{t}\left\|\psi_{x}\right\| L_{L^{\infty}}\left\|\varphi_{x}\right\|\left\|\psi_{x x}\right\| d \tau \\
& \leq \epsilon \int_{0}^{t}\left\|\psi_{x x}\right\|^{2} d \tau+C \epsilon^{-1} \int_{0}^{t}\left\|\psi_{x}\right\|^{2}\left\|V_{x}\right\|^{4} d \tau+C \int_{0}^{t}\left\|\psi_{x}\right\|^{1 / 2}\left\|\varphi_{x}\right\|\left\|\psi_{x x}\right\|^{3 / 2} d \tau \\
& \leq C \epsilon \int_{0}^{t}\left\|\psi_{x x}\right\|^{2} d \tau+C\left(\delta_{0}\right) \int_{0}^{t}\left\|\psi_{x}\right\|^{2} d \tau \\
& \quad+C \epsilon^{-1} \sup _{t}\left\|\varphi_{x}\right\|^{4} \int_{0}^{t}\left\|\psi_{x}\right\|^{2} d \tau .
\end{aligned}
$$

Next we use the definition of $(V, U, \Theta)$ in (1.10), (1.13), Cauchy-Schwarz inequality and (2.8), (2.9), (4.1) to get

$$
\begin{aligned}
& \int_{0}^{t} \int_{0}^{\infty}\left|I_{2}\right| d x d \tau \\
& \quad \leq C \int_{0}^{t} \int_{0}^{\infty}\left(\left|U_{x x}\right||\varphi|+\left|U_{x}\left\|\varphi_{x}|+| U_{x}\right\| V_{x}\left\|\varphi|+| U_{x}\right\| \varphi \| \varphi_{x}\right|\right)\left|\psi_{x x}\right| d x d \tau \\
& \quad \leq \epsilon \int_{0}^{t}\left\|\psi_{x x}\right\|^{2} d \tau+\frac{C}{\epsilon} \int_{0}^{t}\|\varphi\|_{L^{\infty}}^{2}\left\|U_{x x}\right\|^{2} d \tau+\frac{C}{\epsilon} \int_{0}^{t}\left\|U_{x}\right\|_{L^{\infty}}^{2}\left\|\varphi_{x}\right\|^{2} d \tau
\end{aligned}
$$




$$
\begin{aligned}
& +\frac{C}{\epsilon} \int_{0}^{t}\|\varphi\|_{L^{\infty}}^{2}\left\|V_{x}\right\|^{2}\left\|U_{x}\right\|_{L^{\infty}}^{2} d \tau+\frac{C}{\epsilon} \int_{0}^{t}\|\varphi\|_{L^{\infty}}^{2}\left\|U_{x}\right\|_{L^{\infty}}^{2}\left\|\varphi_{x}\right\|^{2} d \tau \\
\leq & \epsilon \int_{0}^{t}\left\|\psi_{x x}\right\|^{2} d \tau+C\left(\delta_{0}\right)+C\left(\delta_{0}\right) \int_{0}^{t}\left\|\varphi_{x}\right\|^{2} d \tau .
\end{aligned}
$$

The same as (4.12) and (4.13), we the definition of $F$ in(1.14) and (2.6) $-(2.8)$, (4.1) we can get the estimates about $I_{3}$ to $I_{5}$ as following.

$$
\begin{aligned}
& \int_{0}^{t} \int_{0}^{\infty}\left(\left|I_{3}\right|+\left|I_{4}\right|+\left|I_{5}\right|\right) d x d \tau \\
& \leq C \int_{0}^{t} \int_{0}^{\infty}\left(\left|\Theta_{x}\right||\varphi|+|\Theta|\left|\varphi_{x}\right|+|\Theta|\left|V_{x}\|\varphi|+| \Theta|| \varphi\| \varphi_{x}\right|\right)\left|\psi_{x x}\right| d x d \tau \\
& \quad+C \int_{0}^{t} \int_{0}^{\infty}\left(\left|\zeta_{x}\right|+|\zeta|\left|V_{x}\right|+|\zeta|\left|\varphi_{x}\right|\right)\left|\psi_{x x}\right| d x d \tau \\
& \quad+\epsilon \int_{0}^{t}\left\|\psi_{x x}\right\|^{2} d \tau+\frac{C}{\epsilon} \int_{0}^{t}\|F\|^{2} d \tau \\
& \leq \epsilon \int_{0}^{t}\left\|\psi_{x x}\right\|^{2} d \tau+C \epsilon^{-1} \int_{0}^{t}\left\|\varphi_{x}\right\|^{2} d \tau+\frac{C}{\epsilon} \int_{0}^{t}\left\|\varphi_{x}\right\|^{2} d \tau+\frac{C}{\epsilon} \int_{0}^{t} \int_{0}^{\infty} V_{x}^{2} \varphi^{2} d x d \tau \\
& \quad+\epsilon \int_{0}^{t}\left\|\psi_{x x}\right\|^{2} d \tau+\frac{C}{\epsilon} \int_{0}^{t} \int_{0}^{\infty}\left(\zeta_{x}^{2}+V_{x}^{2} \zeta^{2}\right) d x d \tau+\frac{C}{\epsilon} \sup _{t}\|(\varphi, \zeta)\|\left\|\left(\varphi_{x}, \zeta_{x}\right)\right\| \int_{0}^{t}\left\|\varphi_{x}\right\|^{2} d \tau \\
& \quad+\epsilon \int_{0}^{t}\left\|\psi_{x x}\right\|^{2} d \tau+C\left(\delta_{0}\right)+C \varphi_{0}(0) .
\end{aligned}
$$

Because Lemma 4.1 and Lemma 4.2, we know $\|(\varphi, \zeta)\|$ is suitably small when $C\left(\delta_{0}\right)$ and $\left\|\left(\varphi_{0}, \psi_{0}, \zeta_{0}\right)\right\|$ small. So there exist a small constant $\delta$ about $\left\|\left(\varphi_{0}, \psi_{0}, \zeta_{0}\right)\right\|$ and $\delta_{0}$ such that

$$
\frac{C}{\epsilon} \sup _{t}\|(\varphi, \zeta)\|\left\|\left(\varphi_{x}, \zeta_{x}\right)\right\| \int_{0}^{t}\left\|\varphi_{x}\right\|^{2} d \tau \leq C \delta \int_{0}^{t}\left\|\varphi_{x}\right\|^{2} d \tau
$$

here $\left\|\varphi_{x}\right\|^{2}+\int_{0}^{t}\left\|\varphi_{x}\right\|^{2} d \tau \leq C$ can be established in Lemma 4.4, Therefore

$$
\begin{aligned}
& \int_{0}^{t} \int_{0}^{\infty}\left(\left|I_{3}\right|+\left|I_{4}\right|+\left|I_{5}\right|\right) d x d \tau \\
& \leq \epsilon \int_{0}^{t}\left\|\psi_{x x}\right\|^{2} d \tau+\frac{C}{\epsilon} \int_{0}^{t} \int_{0}^{\infty}\left(\zeta_{x}^{2}+\varphi_{x}^{2}\right) d x d \tau+C\left(\delta_{0}\right)+C \varphi_{0}(0) .
\end{aligned}
$$

At last we integrate by part to the term about $I_{6}$. Because $\psi_{x}=\varphi_{t}$,

$$
\psi_{t} \psi_{x}(0, t)=\psi_{t} \varphi_{t}(0, t)=\left(\psi \varphi_{t}\right)_{t}(0, t)-\varphi_{0}(0) \frac{p_{+}^{2}}{\mu^{2}} \psi(0, t) e^{-p_{+} t / \mu}
$$

and $\psi(0, t)=C(\ln \Theta)_{x}(0, t)$,

we have

$$
\begin{aligned}
& \left|\int_{0}^{t} \int_{0}^{\infty} I_{6} d x d \tau\right|=\left|\int_{0}^{t}\left(\psi_{t} \varphi_{t}\right)(0, \tau) d \tau\right| \\
& \leq C\left|\psi(0, t) \varphi_{t}(0, t)\right|+C\left|\psi(0,0) \varphi_{t}(0,0)\right|+C \int_{0}^{t} \varphi_{0}(0)|\psi|(0, t) e^{-p_{+} \tau / \mu} d \tau
\end{aligned}
$$




$$
\leq C\left(\delta_{0}\right)
$$

In all we can get

$$
\begin{aligned}
& \int_{0}^{t} \int_{0}^{\infty} \sum_{i=1}^{6}\left|I_{i}\right| d x d \tau \\
& \leq C \int_{0}^{t} \epsilon\left\|\psi_{x x}\right\|^{2} d \tau+C N^{4}(t) \epsilon^{-1} \int_{0}^{t}\left\|\psi_{x}\right\|^{2} d \tau \\
& \quad+C \epsilon^{-1} \int_{0}^{t}\left\|\left(\varphi_{x}, \psi_{x}, \zeta_{x}\right)\right\|^{2} d \tau+C\left(\delta_{0}\right)+C \varphi_{0}(0) .
\end{aligned}
$$

So (4.11) can be change to

$$
\begin{gathered}
\left\|\psi_{x}(t)\right\|^{2}+\int_{0}^{t}\left\|\psi_{x x}(\tau)\right\|^{2} d \tau \leq C\left(\delta_{0}\right)+C \varphi_{0}(0)+C \epsilon^{-1} \int_{0}^{t}\left\|\left(\varphi_{x}, \psi_{x}, \zeta_{x}\right)\right\|^{2} d \tau \\
+C N^{4}(t) \epsilon^{-1} \int_{0}^{t}\left\|\psi_{x}\right\|^{2} d \tau+C\left\|\psi_{0 x}\right\|^{2}
\end{gathered}
$$

The estimate about $\left\|\zeta_{x}\right\|$ is similar to $\left\|\psi_{x}\right\|$, use (3.2) $)_{3}$ multiply $\zeta_{x x}$ then integrate in $Q_{t}=$ $\mathbb{R}_{+} \times(0, t)$ to get

$$
\begin{aligned}
& \left\|\zeta_{x}\right\|^{2}+\int_{0}^{t}\left\|\zeta_{x x}\right\|^{2} d \tau \\
& \leq C\left\|\zeta_{0 x}\right\|^{2}+C \epsilon^{-1} \int_{0}^{t} \int_{0}^{\infty}\left(\psi_{x}^{2}+\zeta \psi_{x}^{2}+\zeta^{2} U_{x}^{2}+U_{x}^{2} \varphi^{2}\right) d x d \tau \\
& \quad+C \int_{0}^{t} \int_{\mathbb{R}_{+}}\left|\zeta_{x}\right|\left(\left|\varphi_{x}\right|+\left|V_{x}\right|\right)\left|\zeta_{x x}\right| d x d \tau+C \epsilon^{-1} \int_{0}^{t} \int_{0}^{\infty}\left|\left(\frac{\Theta_{x} \varphi}{v V}\right)_{x}\right|^{2} d x d \tau \\
& \quad+C \epsilon^{-1} \int_{0}^{\infty} \int_{0}^{\infty}\left(U_{x}^{4}+\psi_{x}^{4}\right) d x d \tau+C \epsilon^{-1} \int_{0}^{t}\|G\|^{2} d \tau \\
& =: C\left\|\zeta_{0 x}\right\|^{2}+\sum_{i=1}^{5} J_{i} .
\end{aligned}
$$

Use the same method as (4.12)-(4.16)

$$
J_{1} \leq C \epsilon^{-1} \int_{0}^{t}\left\|\psi_{x}\right\|^{2} d \tau+C \epsilon^{-1} N^{2}(t) \int_{0}^{t}\left\|U_{x}\right\|^{2} d \tau \leq C \epsilon^{-1} \int_{0}^{t}\left\|\psi_{x}\right\|^{2} d \tau+C\left(\delta_{0}\right) .
$$

Again use the same method as(4.12)-(4.16)

$$
\begin{aligned}
J_{2} & \leq C \int_{0}^{t}\left\|\zeta_{x}\right\|_{L^{\infty}}\left\|\varphi_{x}\right\|\left\|\zeta_{x x}\right\| d \tau+C \int_{0}^{t}\left\|V_{x}\right\|\left\|\zeta_{x}\right\|_{L^{\infty}}\left\|\zeta_{x x}\right\| d \tau \\
& \leq C \int_{0}^{t}\left\|\zeta_{x}\right\|^{1 / 2}\left\|\zeta_{x x}\right\|^{3 / 2}\left\|\varphi_{x}\right\| d \tau+\epsilon \int_{0}^{t}\left\|\zeta_{x x}\right\|^{2} d \tau+C\left(\delta_{0}\right) \int_{0}^{t}\left\|\zeta_{x}\right\|^{2} d \tau \\
& \leq 2 \epsilon \int_{0}^{t}\left\|\zeta_{x x}\right\|^{2} d \tau+C\left(\delta_{0}\right) \int_{0}^{t}\left\|\zeta_{x}\right\|^{2} d \tau+C \epsilon^{-1} \sup _{t}\left\|\varphi_{x}\right\|^{4} \int_{0}^{t}\left\|\zeta_{x}\right\|^{2} d \tau .
\end{aligned}
$$


Because

$$
\begin{aligned}
& \left|\left(\frac{\Theta_{x} \varphi}{v V}\right)_{x}\right|^{2} \\
& =\left|\frac{\Theta_{x x} \varphi}{v V}+\frac{\Theta_{x} \varphi_{x}}{v V}+\frac{\Theta_{x} \varphi}{v V}\left(-\frac{V_{x}+\varphi_{x}}{v^{2}}-\frac{V_{x}}{V^{2}}\right)\right|^{2} \\
& \leq C \Theta_{x x}^{2} \varphi^{2}+C \Theta_{x}^{2} \varphi_{x}^{2}+C \Theta_{x}^{2} V_{x}^{2} \varphi^{2}+C \Theta_{x}^{2} \varphi^{2} \varphi_{x}^{2},
\end{aligned}
$$

combine with $R \Theta / V=p_{+}$, use the same method as (4.12)-(4.16) to get

$$
\begin{aligned}
J_{3} \leq C \epsilon^{-1} \int_{0}^{t}\|\varphi\|_{L^{\infty}}^{2}\left\|\Theta_{x x}\right\|^{2} d \tau+C \epsilon^{-1} \int_{0}^{t}\left\|\Theta_{x}\right\|_{L^{\infty}}^{2}\left\|\varphi_{x}\right\|^{2} d \tau \\
\quad+C \epsilon^{-1} \int_{0}^{t} \int_{0}^{\infty} \Theta_{x}^{2} V_{x}^{2} \varphi^{2} d x d \tau \\
\leq C\left(\delta_{0}\right) \int_{0}^{t}\left\|\varphi_{x}\right\|^{2} d \tau+C\left(\delta_{0}\right)+C \varphi_{0}(0) .
\end{aligned}
$$

Use the definition $U$ and similar as (4.12) (4.13) that we combine with Lemma 2.2 to get

$$
\begin{aligned}
J_{4} & \leq C\left(\delta_{0}\right)+C \epsilon^{-1} \int_{0}^{t}\left\|\psi_{x}\right\|_{L^{\infty}}^{2}\left\|\psi_{x}\right\|^{2} d \tau \\
& \leq C\left(\delta_{0}\right)+C \epsilon^{-1} \int_{0}^{t}\left\|\psi_{x}\right\|^{3}\left\|\psi_{x x}\right\| d \tau \\
& \leq C\left(\delta_{0}\right)+C \int_{0}^{t}\left(\epsilon^{-2}\left\|\psi_{x}\right\|^{2}\left\|\psi_{x}\right\|^{4}+\epsilon^{2}\left\|\psi_{x x}\right\|^{2}\right) d \tau .
\end{aligned}
$$

Use the definition $G$ in (1.14) combine with Lemma 2.2

$$
J_{5}=C \epsilon^{-1} \int_{0}^{t}\|G\|^{2} d \tau \leq C\left(\delta_{0}\right) .
$$

Use the results from $J_{1}$ to $J_{5}$, the inequality (4.19) can be change to

$$
\begin{aligned}
& \left\|\zeta_{x}\right\|^{2}+\int_{0}^{t}\left\|\zeta_{x x}\right\|^{2} d \tau \\
& \leq C\left\|\zeta_{0 x}\right\|^{2}+C\left(\epsilon^{-3}+N^{4}(t)\right) \int_{0}^{t}\left\|\left(\psi_{x}, \zeta_{x}\right)\right\|^{2} d \tau+C\left(\delta_{0}\right) \int_{0}^{t}\left\|\varphi_{x}\right\|^{2} d \tau+C\left(\delta_{0}\right) \\
& \quad+C \epsilon \int_{0}^{t}\left\|\psi_{x x}\right\|^{2} d \tau+C \varphi_{0}(0) .
\end{aligned}
$$

In fact when combine with (4.18) and (4.20), it is easy to get

$$
\begin{aligned}
& \|(\varphi, \psi, \zeta)\|^{2}+\left\|\left(\psi_{x}, \zeta_{x}\right)\right\|^{2}+\int_{0}^{t}\left\|\left(\psi_{x x}, \zeta_{x x}\right)\right\|^{2} d \tau \\
& \leq C\left(\left\|\left(\psi_{0 x}, \zeta_{0 x}\right)\right\|^{2}+\epsilon^{-3}\left\|\left(\varphi_{0}, \psi_{0}, \zeta_{0}\right)\right\|^{2}\right)+C \epsilon^{-1} \int_{0}^{t}\left\|\varphi_{x}\right\|^{2} d \tau+C\left(\delta_{0}\right)+C \varphi_{0}(0) .
\end{aligned}
$$


Lemma 4.4 For a small $\epsilon_{3}>0$ and $C\left(\delta_{0}\right)>0$ is a small constant about $\delta_{0}, C\left(\delta_{0}\right)>0$ is a small constant about $\delta_{0}$, we can get

$$
\left\|\varphi_{x}\right\|^{2}+\int_{0}^{t}\left\|\varphi_{x}\right\|^{2} d \tau \leq C\left\|\varphi_{0 x}\right\|^{2}+C \epsilon_{3}^{-1}\left\|\left(\varphi_{0}, \psi_{0}, \zeta_{0}\right)\right\|^{2}+C\left(\delta_{0}\right)+C \varphi_{0}(0) .
$$

Proof. Set $\bar{v}=\frac{v}{V}$ take it into $(\underline{3.2})_{1},(\underline{(3.2})_{2}(p=R \theta / v)$ to get

$$
\psi_{t}+p_{x}=\mu\left(\frac{\bar{v}_{x}}{\bar{v}}\right)_{t}-F
$$

Both sides of last equation multiply $\bar{v}_{x} / \bar{v}$ to get

$$
\begin{aligned}
& \left(\frac{\mu}{2}\left(\frac{\bar{v}_{x}}{\bar{v}}\right)^{2}-\psi \frac{\bar{v}_{x}}{\bar{v}}\right)_{t}+\frac{R \theta}{v}\left(\frac{\bar{v}_{x}}{\bar{v}}\right)^{2}+\left(\psi \frac{\bar{v}_{t}}{\bar{v}}\right)_{x} \\
& \quad=\frac{\psi_{x}^{2}}{v}+U_{x}\left(\frac{1}{v}-\frac{1}{V}\right) \psi_{x}+\frac{R \zeta_{x}}{v} \frac{\bar{v}_{x}}{\bar{v}}-\frac{R \theta}{v}\left(\frac{1}{\Theta}-\frac{1}{\theta}\right) \Theta_{x} \frac{\bar{v}_{x}}{\bar{v}}+F \frac{\bar{v}_{x}}{\bar{v}} .
\end{aligned}
$$

On the boundary condition we have

$$
\psi(0, \tau) \frac{\bar{v}_{\tau}(0, \tau)}{\bar{v}(0, \tau)}=\psi(0, \tau) \frac{\varphi_{\tau}(0, \tau)}{v(0, \tau)}
$$

When use the definition $\psi(0, t)$ in (3.2) and $\varphi_{t}(0, t)$ in (4.3) we can get

$$
\int_{0}^{t}\left|\psi(0, \tau) \frac{\bar{v}_{\tau}(0, \tau)}{\bar{v}(0, \tau)}\right| d \tau \leq C\left(\delta_{0}\right)
$$

On the other hand if we integrate (4.22) in $R_{+} \times(0, t)$, (4.22) is changed to

$$
\begin{aligned}
& \int_{\mathbb{R}_{+}}\left(\frac{\mu}{2}\left(\frac{\bar{v}_{x}}{\bar{v}}\right)^{2}-\psi \frac{\bar{v}_{x}}{\bar{v}}\right) d x-\int_{\mathbb{R}_{+}}\left(\frac{\mu}{2}\left(\frac{\bar{v}_{x}(x, 0)}{\bar{v}(x, 0)}\right)^{2}-\psi_{0} \frac{\bar{v}_{x}(x, 0)}{\bar{v}(x, 0)}\right) d x \\
& \quad+\int_{0}^{t} \int_{\mathbb{R}_{+}}\left(\frac{R \theta}{v}\left(\frac{\bar{v}_{x}}{\bar{v}}\right)^{2}+\left(\psi \frac{\bar{v}_{t}}{\bar{v}}\right)_{x}\right) d x d \tau \\
& \leq C \epsilon^{-1}\left(\int_{0}^{t}\left\|\left(\zeta_{x}, \psi_{x}\right)\right\|^{2} d \tau+\int_{0}^{t} \int_{0}^{\infty} \Theta_{x}^{2}\left(\varphi^{2}+\zeta^{2}\right) d x d \tau\right) \\
& +C \epsilon^{-1} \int_{0}^{t} \int_{0}^{+\infty} U_{x}^{2} \varphi^{2} d x d \tau+C \epsilon^{-1} \int_{0}^{t} \int_{0}^{+\infty}|F|^{2} d x d \tau+\epsilon \int_{0}^{t}\left\|\frac{\bar{v}_{x}}{\bar{v}}\right\|^{2} d \tau+C\left(\delta_{0}\right) .
\end{aligned}
$$

Insert (4.23) into (4.24) and combine with the definition of $F$ and $U$ and Lemma 2.2, we can get

$$
\begin{aligned}
& \int_{0}^{t}\left\|\frac{\bar{v}_{x}}{\bar{v}}\right\|^{2} d \tau+\left\|\frac{\bar{v}_{x}}{\bar{v}}\right\|^{2}-C \epsilon^{-1}\|\psi\|^{2}-C\left\|\psi_{0}\right\|^{2}-C \int_{0}^{\infty} \frac{\bar{v}_{x}}{\bar{v}}(x, 0)^{2} d x \\
& \leq C \epsilon^{-1}\left(\int_{0}^{t}\left\|\left(\zeta_{x}, \psi_{x}\right)\right\|^{2} d \tau+\int_{0}^{t} \int_{0}^{\infty} \Theta_{x}^{2}\left(\varphi^{2}+\zeta^{2}\right) d x d \tau+C\left(\delta_{0}\right)\right) \\
& \quad+\epsilon \int_{0}^{t}\left\|\frac{\bar{v}_{x}}{\bar{v}}\right\|^{2} d \tau+C\left\|\varphi_{0 x}\right\|^{2} .
\end{aligned}
$$


Because $C_{1}\left(\varphi_{x}^{2}\right)-C_{2} V_{x}^{2} \leq\left(\frac{\bar{v}_{x}}{\bar{v}}\right)^{2} \leq C_{3} \varphi_{x}^{2}+C_{4} V_{x}^{2}\left(C_{1}, C_{2}, C_{3}, C_{4}\right.$ stands for constants about $\left.v\right)$, combine with Lemma 4.1 - 4.2 we change (4.25) to

$$
\int_{0}^{t}\left\|\varphi_{x}\right\|^{2} d \tau+\left\|\varphi_{x}\right\|^{2} \leq C\left\|\varphi_{0 x}\right\|^{2}+\int_{0}^{t} C \epsilon_{3}^{-1}\left\|\left(\psi_{x}, \zeta_{x}\right)\right\|^{2} d \tau+C\left(\delta_{0}\right)+C \varphi_{0}(0) .
$$

So we finish this lemma.

From Lemma 4.2 to Lemma 4.4 we know when $\delta_{0}$ and $\left\|\left(\varphi_{0}, \psi_{0}, \zeta_{0}\right)\right\|$ suitably small there exist a suitably small constant $\delta$ such that

$$
\|(\varphi, \psi, \zeta)\|^{2}+\int_{0}^{t}\left\|\left(\psi_{x}, \zeta_{x}\right)\right\|^{2} d \tau \leq C \delta
$$

and

$$
\left\|\left(\varphi_{x}, \psi_{x}, \zeta_{x}\right)\right\|^{2}+\int_{0}^{t}\left\|\left(\psi_{x x}, \zeta_{x x}\right)\right\|^{2} \leq C .
$$

Then we can get $C_{5} \leq|v| \leq C_{6}$ and $C_{7} \leq|\theta| \leq C_{8}$ when $\delta$ small, here $C_{5}, C_{6}, C_{7}$ and $C_{8}$ are constants independent of $v$ and $\theta$. So we can get (3.3) in Proposition 2.2 .

To finish Theorem 3.1 now we will proof $\sup _{x \in \mathbb{R}_{+}}|(\varphi, \psi, \zeta)| \rightarrow 0$, as $t \rightarrow \infty$.

Because $\int_{0}^{\infty} \partial_{x}(\underline{(3.2)})_{1} \times 2 \varphi_{x} d x$ equals to

$$
0=2 \int_{0}^{\infty} \varphi_{x} \psi_{x x} d x-\frac{d}{d t}\left\|\varphi_{x}\right\|^{2}
$$

use Cauchy-Schwarz inequality we get

$$
2 \int_{0}^{\infty} \varphi_{x} \psi_{x x} d x \leq C\left(\left\|\varphi_{x}\right\|^{2}+\left\|\psi_{x x}\right\|^{2}\right)
$$

Again using Lemma 4.34 .4 , we get

$$
\begin{aligned}
& \int_{0}^{\infty}\left|\frac{d}{d t}\left\|\varphi_{x}(t)\right\|^{2}\right| d t \\
& \leq C \int_{0}^{\infty}\left(\left\|\varphi_{x}\right\|^{2}+\left\|\psi_{x x}\right\|^{2}\right) d t \\
& \leq C\left(C\left(\delta_{0}\right)+\left\|\left(\varphi_{0}, \psi_{0}, \zeta_{0}\right)\right\|_{1}^{2}+C \varphi_{0}(0)\right) .
\end{aligned}
$$

Similar as above, from Lemma 4.2 4.4 and combine with Sobolev inequality we get

$$
\int_{0}^{\infty}\left(\left|\frac{d}{d t}\left\|\psi_{x}(t)\right\|^{2}\right|+\left|\frac{d}{d t}\left\|\zeta_{x}(t)\right\|^{2}\right|\right) d \tau \leq C\left(C\left(\delta_{0}\right)+\left\|\left(\varphi_{0}, \psi_{0}, \zeta_{0}\right)\right\|_{1}^{2}+C \varphi_{0}(0)\right) .
$$

It means

$$
\|(\varphi, \psi, \zeta)(t)\|_{L^{\infty}}^{2} \leq 2\|(\varphi, \psi, \zeta)(t)\|\left\|\left(\varphi_{x}, \psi_{x}, \zeta_{x}\right)(t)\right\| \rightarrow 0 \quad \text { when } \quad t \rightarrow \infty .
$$

So we finish Theorem $3.1, \square$ 


\section{References}

[1] Z.P. Xin, On nonlinear stability of contact discontinuities. Proceeding of 5th International Conferences on Hyperbolic Problems: Theory, Numerics and Applications. Ed. Glimm, etc., World Sci. Publishing, River Edge, NJ, 1996.

[2] T.P. Liu, Z.P. Xin, Pointwise decay to contact discontinuities for systems of viscous conservation laws. Asian J. Math., 1 (1997) 34-84.

[3] F.M. Huang, A. Matsumura, X. Shi, On the stability of contact discontinuity for compressible Navier-Stokes equations with free boundary. Osaka J. Math., 41 (1) (2004) 193-210.

[4] F.M. Huang, A. Matsumura, Z.P.Xin, Stability of contact discontinuity for the 1-D compressible Navier-Stokes Equations. Arch. Ratonal Mech. Anal., 179 (2005) 55-77.

[5] F.M. Huang, Z.P. Xin, T.Yang, Contact discontinuity with general perturbations for gas motions. Adv. Math., 219 (2008) 1246-1297.

[6] F.M. Huang, H.J. Zhao, On the global stability of contact discontinuity for compressible Navier-Stokes equations. Rend. Sem. Mat. Univ. Padova, 109 (2003) 283-305.

[7] F.M. Huang, J. Li, A. Matsumura, Asymptotic stability of combination of viscous contact wave with rarefaction waves for one-dimensional compressible Navier-Stokes system. Arch.Ration.Mech.Anal., 197(1)(2010) 89-116 .

[8] Ma S. X. ,Zero dissipation limit to strong contact discontinuity for the $1-D$ compressible Navier-Stokes equations, JDE., 248(2010) 95-110.

[9] Hakho Hong, Global stabillity of viscous contact wave for $1-D$ compressible Navier-Stokes equations, JDE., 252(2012) 3482-3505.

[10] A. Matsumura, Inflow and outflow problems in the half space for a one-dimensional isentropic model system of compressible viscous gas. Meth. Appl. Anal., 8(4) (2001) 645-666.

[11] T.Zheng, JW. Zhang, JN.Zhao, Asymptotic stability of viscous contact discontinuity to an inflow problem for compressible NavierCStokes equations,Nonlinear Analysis: Nonlinear Anal. Theor., 74(17)(2011) 6617-6639.

[12] T.Zheng and J.Zhao, On the stability of contact discontinuity for Cauchy problem of compress Navier-Stokes equations with general initial data. Sci. China. Math., 55(10)(2012) 2005-2026.

[13] K. Nishihara, T. Yang, H.J. Zhao, Nonlinear stability of strong rarefaction waves for compressible Navier-Stokes equations. SIAM J. Math. Anal., 35 (2004) 1561-1597.

[14] Tao Wang;Huijiang Zhao;Qingyang Zou,One-dimensional compressible Navier-Stokes equations with large density oscillation,Kinetic and Related Models, 6(3)(2013)649-670.

[15] S. Kawashima, A. Matsumura, Asymptotic stability of traveling wave solutions of systems for one-dimensional gas motion. Commun. Math. Phys., 101 (1985) 97-127.

[16] S. Kawashima, A. Matsumura, K. Nishihara, Asymptotic behavior of solutions for the equations of a viscous heat-conductive gas. Proc. Japan Acad. Ser. A, 62 (1986) 249-252. 
[17] A. Matsumura, K. Nishihara, Large-time behaviors of solutions to an inflow problem in the half space for a one-dimensional system of compressible viscous gas, Comm.Math.Phys.222(2001)449-474.

[18] S. Kawashima, S. Nishibata, P.C. Zhu, Asymptotic stability of the stationary solution to the compressible Navier-Stokes equations in the half space. Comm. Math. Phys., 240 (2003) 483-500.

[19] S. Kawashima, P.C. Zhu, Asymptotic stability of nonlinear wave for the compressible Navier-Stokes equations in the half space. J. Diff. Eqn., 244 (2008), 3151-3179.

[20] T.P. Liu, Nonlinear stability of shock waves for viscous conservation laws, Mem. Amer.Math.Soc.,56(328),(1985).

[21] A. Matsumura, K. Nishihara, On the stability of travelling wave solutions of a onedimensional model system for compressible viscous gas. Japan J. Appl. Math., 2 (1985) $17-25$.

[22] A. Matsumura, K. Nishihara, Asymptotics toward the rarefaction waves of the solutions of a one-dimensional model system for compressible viscous gas. Japan J. Appl. Math., 3 (1985) 1-13.

[23] A. Matsumura, K. Nishihara, Global stability of the rarefaction wave of a one-dimensional model system for compressible viscous gas. Commun. Math. Phys., 165 (1992) 325-335.

[24] Szepessy,A., Xin, Z.P., Nnlinear stability of viscous shck waves, Arch.Ration.Mech.Anal.,122(1993)53-103.

[25] Szepessy,A., Zumbrun,K., Stability of rarefaction waves in viscous media. Arch.Ration.Mech.Anal., 133(1996)249-298. 